\title{
Estimation of the wave-related ripple characteristics and induced bed shear stress
}

\author{
Benoît Camenen
}

Cemagref, UR HHLY, 3 bis quai Chauveau, CP 220, F-69336 Lyon cedex 09, France

Email: benoit.camenen@cemagref.fr

\begin{abstract}
A large data set on ripples was collected and examined. A set of new formulas for the prediction of the ripple characteristics is proposed with an emphasis on the disappearance of the ripples. The ripple wavelength was observed to be proportional to the bottom wave excursion but also to be a function of the grain-related Shields parameter and wave period parameter introduced by Mogridge et al. (1994). The ripple steepness was found to be nearly constant for orbital ripples, and with a sharp decrease for suborbital ripples. Two empirical functions are added including the effects of the critical Shields parameters (inception of transport and inception of sheet flow), i.e. giving the boundaries for the ripple existence's domain. The proposed formulas yield better prediction capabilities compared to the previously published formulas, especially when ripples are washed out. The effect of the ripple characteristics on the roughness height and the calculation of the bed shear stress is also discussed. It appeared that the bed shear stress calculation is more sensitive to the empirical coefficient $a_{r}$ introduced in the estimation of the ripple-induced roughness height or to the limits of existence of the ripples than the ripple characteristics themselves.
\end{abstract}

\section{Keywords}

wave ripples; ripple height; ripple steepness; roughness height; bed shear stress; predictive formula

\section{Introduction}

An important issue for the prediction of the suspended load in coastal waters is to estimate the bottom shear stress due the oscillatory movement of the waves. Close to the shore, the wave-related bed shear stress often prevails on the current-related bed shear stress, and thus significantly influences the estimation of the bottom reference concentration and sediment diffusivity, which are the main parameters for 
the suspended load (Camenen \& Larson, 2008). When ripples appear, their effects on the roughness height and bottom shear stress are very significant (Van Rijn, 1993). Van der Werf et al. (2006) also showed the strong influence of ripples on the direction of the net suspended load, as they induce some phase-lag in the suspension concentration. It is then extremely important to predict correctly the characteristics of the ripples and induced roughness height.

Unlike current-related bed forms, wave ripples are generally symmetric with sharper crests due to the to and fro movement of wave orbital velocity. Bagnold (1946) called rolling-grain ripples the ripples first appearing on a flat bed exposed to wave-action. For this regime close to the inception of motion, the grains roll back and forth and form small triangular ridges. According to Bagnold (1946), these ripples are stable only if the shear stress stays close to its critical value for the inception of movement. If the flow magnitude increases, the lee-side vortex becomes strong enough to initiate grain motion in the space between the two ripples. The ripples grow until an equilibrium geometry corresponding to the so-called vortex ripples.

The ripple wave length $L_{r}$ is often assumed to be proportional to the semiexcursion amplitude of orbital motion $A_{w}: 1<L_{r} / A_{w}<2$. And the ripple wave steepness $H_{r} / L_{r}$ (where $H_{r}$ is the ripple height) varies from 0.1 (vortex formation) to 0.25 (slope stability). Wiberg \& Harris (1994) made a very careful description of ripple characteristics. In their classification, ripples are subdivided into three groups: orbital, suborbital and anorbital. The basic scaling of orbital ripple wavelength is on $A_{w}$, whereas the basic scaling of anorbital ripples is on the median grain size $d_{50}$; suborbital ripples represent the transitional region between these two limits. Orbital ripples have an almost constant steepness of $H_{r} / L_{r}=0.17$, while anorbital ripples are less steep, with a steepness decreasing as orbital diameter increases for a given grain size.

Recently, Hanes et al. (2001) observed in the field long-wave ripples (LWR) with relatively small heights (ranging from $3 \mathrm{~mm}$ to $6 \mathrm{~cm}$ ) and large lengths (ranging from 35 to $200 \mathrm{~cm}$ ). LWR were observed to be not as dynamics as the small-wave ripples (SWR) commonly observed. And they also observed some coexistence for both types of ripples. One explanation for LWR is that the peak frequency near the seabed may be different (smaller) than the peak frequency of the surface elevation because of the frequency-dependent reduction of fluid orbital amplitude with depth. Other assumptions for their origin may be a larger suspension within the boundary layer and a regime close to the sheet-flow regime (Hanes et al., 2001). Kleinhans (2005) described them as skewed hummocks with much sediment suspension but no strong vortex shedding, associated with large orbital flows possibly combined with small currents.

For strong conditions (typically storm conditions), ripples are washed out. It corresponds to the inception of the sheet-flow regime where most of the sediment transport occurs in a thin layer close to the bed. This regime occurs when the Shields parameter, mobility parameter, orbital Reynolds number or the ratio $A_{/} d_{50}$ reach some critical value: $\theta_{w}>0.8, \Psi_{w}>100, \Re_{w}>2 \times 10^{6}$ or $A / d_{50}=6000$ approximately ( $c f$. Van Rijn, 1993 or Camenen \& Larson, 2006) where the wave- 
related Shields parameter, mobility parameters, wave period parameter, and orbital Reynolds number are defined as follows:

$$
\begin{aligned}
\theta_{w} & =\frac{0.5 f_{w} U_{w}{ }^{2}}{(s-1) g d_{50}} \\
\Psi_{w} & =\frac{U_{w}{ }^{2}}{(s-1) g d_{50}}=\left[\frac{\pi A_{w}}{d_{50}}\right]^{2} \chi \\
\chi & =\frac{d_{50}}{(s-1) g T_{w}{ }^{2}} \\
\Re_{w} & =\frac{U_{w}{ }^{2}}{\omega \nu}
\end{aligned}
$$

with $U_{w}$ the wave orbital velocity, $s$ the relative sediment density, $g$ the acceleration of gravity, $f_{w}$ the wave-related friction factor, $A_{w}=U_{w} T_{w} /(2 \pi)$ the wave orbital semi excursion, $T_{w}$ the wave period, $\omega=2 \pi / T_{w}$, and $\nu$ the kinematic viscosity of water.

Many authors proposed empirical relationships to describe the ripple characteristics, based on the mobility parameter $\Psi_{w}$ : Nielsen (1981), Wikramanayake \& Madsen (1991), Van Rijn (1993), or Grasmeijer \& Kleinhans (2004); on the Shields parameter $\theta_{w}$ : Grant \& Madsen (1982); on the wave period parameter $\chi$ : Mogridge et al. (1994); on the ratio $A_{w} / d_{50}$ : Wiberg \& Harris (1994) or Soulsby \& Whitehouse (2005 and 2007?); or on the orbital Reynolds number: Williams et al. (2005). These equations are presented in detail in appendix. Most of these formulas are however only based on experimental data where ripples exist. In order to properly estimate the total shear stress, it is also fundamental to validate these formulas over plane beds when the ripples are washed out. For random waves, authors usually suggested to use the significant wave characteristics for the prediction. Then, most of the formulas are assumed valid for both monochromatic and random waves.

The main purpose of this study is to provide a formula which correctly predicts ripple characteristics but also ripple disappearance in order to estimate properly the total bed shear stress for any wave condition. This paper is organized as follows: The experimental data used for this study are described and compared with the studied formulas. New formulas for the prediction of the ripple characteristics and their validation are presented next. Then, a presentation of the computation of the total shear stress is made as well as a sensitivity analysis on the main parameters. Finally, conclusions concerning the validity of the formulas and a discussion on the calculation of the total shear stress in the nearshore are also provided.

\section{On the prediction of ripple characteristics}

\section{$2.1 \quad$ Experimental data}

In order to compare the results obtained using empirical formulas with experimental data, several data sets were compiled. In Tab. 1, the main hydrodynamic and sedimentologic parameters are presented, as well as the type of the experiment. 
Table 1: Data summary for experiments on ripple characteristics.

\begin{tabular}{|c|c|c|c|c|c|c|c|c|c|c|c|}
\hline Author(s) & Name & Location & $\begin{array}{l}\text { Flow } \\
\text { Type }\end{array}$ & $\begin{array}{l}\text { nbr. } \\
\text { exp. }\end{array}$ & $\begin{array}{c}d_{50} \\
{[m m]}\end{array}$ & $\begin{array}{c}\boldsymbol{h} \\
{[m]}\end{array}$ & $\begin{array}{c}\boldsymbol{U}_{\boldsymbol{c}} \\
{[\mathrm{m} / \mathrm{s}]}\end{array}$ & $\begin{array}{c}\boldsymbol{U}_{\boldsymbol{w}} \\
{[\mathrm{m} / \mathrm{s}]}\end{array}$ & $\begin{array}{l}\boldsymbol{T}_{\boldsymbol{w}} \\
{[s]}\end{array}$ & $\begin{array}{l}\boldsymbol{H}_{r} \\
{[\mathrm{~cm}]}\end{array}$ & $\begin{array}{c}\boldsymbol{L}_{r} \\
{[\mathrm{~cm}]}\end{array}$ \\
\hline Inman (1957) & Inm57 & US Corps of Engineers, USA & field & 41 & $0.1-0.9$ & $0.5-2.0$ & - & $0.06-0.57$ & $5.0-13$ & $0.5-19$ & $6-105$ \\
\hline Lofquist (1978) & Lof78 & US Corps of Engineers, USA & $\begin{array}{l}\text { Oscillatory } \\
\text { flow tunnel }\end{array}$ & 13 & $0.18-0.26$ & 0.4 & 0 & $0.41-1.22$ & $2.7-15$ & $0-19$ & $11-120$ \\
\hline Sleath (1982) & Sle02 & $\begin{array}{l}\text { Cambridge University, United } \\
\text { Kingdom }\end{array}$ & $\begin{array}{l}\text { oscillatory } \\
\text { flow tunnel }\end{array}$ & 13 & $0.20,0.41$ & 0.15 & 0 & $0.16-0.44$ & $2.9-5.1$ & $2-5$ & $12-32$ \\
\hline $\begin{array}{l}\text { Bosman (1982) and Steet- } \\
\text { zel (1985) }\end{array}$ & DLL80s & DHL, Delft, The Netherlands & wave flume & 70 & 0.10 & $0.1-0.65$ & $0.10-0.32$ & $0.13-0.30$ & $1.4-2.0$ & $1-3$ & 8 \\
\hline Sato (1987) & Sat87 & Tokyo University, Japan & $\begin{array}{l}\text { oscillatory } \\
\text { flow tunnel }\end{array}$ & 66 & 0.18 & 0.21 & 0 & $0.11-0.65$ & $0.5-7.0$ & $0-3$ & $0-18$ \\
\hline $\begin{array}{llll}\begin{array}{l}\text { Ribberink } \\
(1994)\end{array} & \& & \text { Al } & \text { Salem } \\
\end{array}$ & Rib90s & DHL, Delft, The Netherlands & $\begin{array}{l}\text { large water } \\
\text { tunnel }\end{array}$ & 71 & 0.21 & 0.8 & 0 & $0.2-1.5$ & $2.0-12.0$ & $0-35$ & $0-300$ \\
\hline Van Rijn et al. (1993) & VR93 & Delft Univ., The Netherlands & wave flume & 45 & $0.1-0.22$ & $0.48-0.52$ & $-0.45-0.45$ & $0.14-0.39$ & $2.2-2.7$ & $0.6-2.9$ & $6-20$ \\
\hline Van Rijn \& Havinga (1995) & VRH95 & DHL, Delft, The Netherlands & wave basin & 28 & 0.1 & $0.40-0.43$ & $0-0.32$ & $0.14-0.30$ & $2.1-2.3$ & $0.6-1.4$ & $6-11$ \\
\hline Hume et al. (1999) & Hum99 & NIWA, USA & field & 9 & 0.4 & 25 & - & $0.28-1.06$ & 11.0 & $3-13$ & $40-90$ \\
\hline Khelifa \& Ouellet (2000) & KO00 & Laval, Canada & wave basin & 48 & $0.15-0.5$ & 0.3 & $0-0.34$ & $0.08-0.26$ & $0.9-1.4$ & $0.4-1.7$ & $3-12$ \\
\hline Doucette $(2000,2002)$ & Dou02 & Various beaches, Australia & field & 84 & $0.14-0.62$ & $0.18-1.73$ & - & $0.15-1.00$ & $2.2-12.2$ & $0-14$ & $0-91$ \\
\hline SEDMOC data set (2001) & TUF80s & $\begin{array}{l}\text { Grote Speurwerk (35m), DUT, } \\
\text { Delft, The Netherlands }\end{array}$ & wave flume & 125 & $0.10-0.22$ & $0.29-0.60$ & $0.07-0.45$ & $0.17-0.55$ & $1.2-2.7$ & $0.2-2.9$ & $0.6-20$ \\
\hline $\begin{array}{l}\begin{array}{l}\text { O'Donoghue } \\
\text { (2001) }\end{array} \\
\text { \& }\end{array}$ & oDC01 & $\begin{array}{l}\text { Aberdeen University, United } \\
\text { Kingdom }\end{array}$ & flow tunnel & 35 & $0.18-0.44$ & 0.75 & 0 & $0.25-0.94$ & $2.0-15$ & $0.9-19$ & $6-121$ \\
\hline Hanes et al. (2001) & Han01 & Duck, North Carolina, USA & field & 201 & $0.12-1.7$ & $1.4-7$ & - & $0.12-1.62$ & $3.1-16$ & $0-13$ & $0-270$ \\
\hline Sleath \& Wallbridge (2002) & Sle02 & $\begin{array}{l}\text { Cambridge University, United } \\
\text { Kingdom }\end{array}$ & $\begin{array}{l}\text { oscillatory } \\
\text { flow tunnel }\end{array}$ & 29 & $0.20-0.80$ & 0.15 & 0 & $0.12-1.64$ & $2.8-6.8$ & $0-9$ & $0-50$ \\
\hline Faraci \& Foti (2002) & FF02 & Catania University, Italy & wave flume & 38 & 0.25 & 0.29 & - & $0.12-0.35$ & $1.2-4.2$ & $0.7-2.1$ & $4-11$ \\
\hline $\begin{array}{lccc}\begin{array}{l}\text { Delgado } \\
(2004)\end{array} & \text { Blanco } & \text { et } & \text { al. } \\
\end{array}$ & Del04 & $\begin{array}{l}\text { Deltaflume, Delft, The Nether- } \\
\text { lands }\end{array}$ & $\begin{array}{l}\text { large wave } \\
\text { flume }\end{array}$ & 17 & $0.18-0.44$ & 7.0 & 0 & $0.14-0.74$ & 6 & $0.6-5$ & $10-35$ \\
\hline $\begin{array}{l}\text { Van der Werf \& Ribberink } \\
(2004)\end{array}$ & vdWR04 & DHL, Delft, The Netherlands & $\begin{array}{l}\text { large water } \\
\text { tunnel }\end{array}$ & 10 & 0.35 & 0.8 & 0 & $0.42-0.85$ & $5.0-10.0$ & $2.5-13.9$ & $44-113$ \\
\hline $\begin{array}{l}\text { Grasmeijer \& Kleinhans } \\
(2004)\end{array}$ & GK04 & $\begin{array}{l}\text { Egmond aan Zee, The Nether- } \\
\text { lands }\end{array}$ & field & 45 & 0.24 & $1.5-5.0$ & $-0.40-0.10$ & $0.23-0.99$ & $4.0-10.5$ & $0.7-10$ & $19-200$ \\
\hline Williams et al. (2004) & Wil04 & $\begin{array}{l}\text { Deltaflume, Delft, The Nether- } \\
\text { lands }\end{array}$ & $\begin{array}{l}\text { large wave } \\
\text { flume }\end{array}$ & 65 & $0.16-0.35$ & $4.0-4.5$ & 0 & $0.13-1.03$ & $4.0-6.0$ & $0.3-7$ & $20-104$ \\
\hline $\begin{array}{l}\text { Catano-Lopera \& } \text { Garcia } \\
(2006 \mathrm{a}, \mathrm{b})\end{array}$ & Cat06 & University of Illinois, USA & wave flume & 23 & 0.25 & $0.36-0.76$ & $0-0.6$ & $0.25-0.42$ & $2.0-6.9$ & $1.5-3.5$ & $9.1-18.8$ \\
\hline
\end{tabular}


For all the experiments presented in Tab. 1, sand with a relative density $s=$ 2.65 was used. Several of these data sets were obtained from the data compilation provided by the SEDmoc (2001) European Union research project.

\subsection{Comparison between experimental data and empirical relationships for ripple characteristics}

A comparison between the experimental data and the empirical relationships presented previously was undertaken. Tab. 2 summarizes the results obtained for the prediction of the ripple height and length where $P 2$ and $P 5$ indicate the percentage of data correctly predicted within a factor 2 or 5 , respectively. The mean value $\left(\mu_{f}\right)$ and standard deviation $\left(\sigma_{f}\right)$ of the function $f(X)=\log \left|X_{\text {pred }} / X_{\text {meas }}\right|$ is also presented, where $X_{\text {pred }}$ and $X_{\text {meas }}$ are the predicted and measured values $\left(H_{r}\right.$ or $L_{r}$ ), respectively. To avoid zero-values in the experimental data or predicted data, a minimum value for $H_{r}$ and $L_{r}$ was assumed (equal to $2 d_{50}$ and $10 d_{50}$, respectively). The Williams et al. (2005) formulas were proposed for suborbital ripples only and thus are valid for large values of the ratio $A_{w} / d_{50}$ only. Because of its obvious limits, this formula will not be used in the following study.

Table 2: Prediction of the ripple characteristics using various empirical equations and the collected experimental data with purely oscillatory flows.

\begin{tabular}{l|cccc|cccc}
\hline & \multicolumn{4}{|c|}{ ripple length $L_{r}$} & \multicolumn{4}{c}{ ripple height $H_{r}$} \\
Equation & $P 2$ & $P 5$ & $\mu_{f}$ & $\sigma_{f}$ & $P 2$ & $P 5$ & $\mu_{f}$ & $\sigma_{f}$ \\
\hline Nielsen & $35 \%$ & $61 \%$ & -0.11 & 0.84 & $35 \%$ & $60 \%$ & -0.34 & 0.79 \\
Grant \& Madsen & $59 \%$ & $76 \%$ & +0.36 & 0.99 & $44 \%$ & $71 \%$ & +0.17 & 0.85 \\
Wikramanayake \& Madsen & $45 \%$ & $75 \%$ & +0.04 & 0.81 & $37 \%$ & $67 \%$ & -0.09 & 0.74 \\
Van Rijn & $61 \%$ & $80 \%$ & +0.10 & 0.84 & $56 \%$ & $78 \%$ & +0.02 & 0.79 \\
Mogridge et al. & $62 \%$ & $82 \%$ & +0.36 & 0.78 & $45 \%$ & $71 \%$ & +0.63 & 0.82 \\
Wiberg \& Harris & $54 \%$ & $82 \%$ & +0.09 & 0.76 & $55 \%$ & $82 \%$ & +0.15 & 0.66 \\
Grasmeijer \& Kleinhans & $58 \%$ & $81 \%$ & +0.26 & 0.89 & $39 \%$ & $74 \%$ & +0.02 & 0.74 \\
Soulsby \& Whitehouse & $66 \%$ & $79 \%$ & +0.19 & 0.80 & $65 \%$ & $84 \%$ & +0.06 & 0.63 \\
Eqs. 9] and 10 with $\alpha_{c r}=0.01$ & $66 \%$ & $81 \%$ & +0.17 & 0.76 & $68 \%$ & $88 \%$ & -0.05 & 0.56 \\
Eqs. 9 and 10 with $\alpha_{c r}=1$ & $64 \%$ & $80 \%$ & +0.02 & 0.83 & $65 \%$ & $84 \%$ & -0.14 & 0.64 \\
\hline
\end{tabular}

For the prediction of the ripple length, it appears that the Grant \& Madsen, Mogridge et al., Van Rijn, Wiberg \& Harris, Grasmeijer \& Kleinhans formulas and more especially the Soulsby \& Whitehouse formula yield the best results. For the prediction of the ripple height (which is actually dependent on the prediction of the ripple length), the Van Rijn, Wiberg \& Harris, and Soulsby \& Whitehouse formulas yield the best results. Apart from the Nielsen and Wikramanayake \& Madsen formulas, all formulas tend to generally overestimate measured ripple height and length. The Wiberg \& Harris and Mogridge et al. formulas yield smaller dispersion 
for the ripple height prediction compared to the Grant \& Madsen, Soulsby \& Whitehouse and Van Rijn formulas. This may be partly explained as former formulas do not take into account the critical Shields parameters for the presence of ripples. As the comparison is made with data where ripples exist, a model that predicts the absence of ripple would yield significant underestimation. It is particularly significant for the effect of $\theta_{c r}$ as several data indicate the occurrence of ripples whereas the Shields parameter is smaller than $\theta_{c r}$ (estimated in this paper using Eq. 5). Actually, as noted by Soulsby \& Whitehouse (2007?), when $\theta_{w} \leq \theta_{c r}$, ripples should take pre-existing values of $H_{r}$ and $L_{r}$, which are not necessarily null. However, in nature, there are many cases where the bed is flat. Even if some uncertainties exist on the prediction on the critical Shields parameters (inception of sediment movement or sheet flow), it is very important to be able to predict the appearance and disappearance of ripples and not to overestimate the roughness height when the bed is flat. The Nielsen, Van Rijn and Grasmeijer \& Kleinhans formulas give a sheet flow threshold for $\left(\theta_{w}\right)_{c r, s f}=1$ or $\left(\Psi_{w}\right)_{c r, s f}=250$ or 156 , respectively. Another remark is that the Wiberg \& Harris and Soulsby \& Whitehouse formulas predict a nearly constant value for the ratio $H_{r} / L_{r} \approx 0.15$ when $A_{w} / d_{50}<1000$ and a rapid decrease in steepness when $A_{w} / d_{50}>3000$. For these two formulas, the threshold of sheet-flow is described in term of $A_{w} / d_{50}\left(\left(A_{w} / d_{50}\right)_{c r, s f} \approx 6000\right.$, which seems to be too large, see section 3.1). As a conclusion, the Soulsby \& Whitehouse formula yields the best prediction for the estimation of the ripple characteristics using the collected data; the Van Rijn formula yields a lower score for the prediction but it seems to better take into account the effect of the threshold for the inception of sheet flow.

\subsection{Capabilities of the various formulas to predict the ab- sence of ripples}

To confirm these observations, a comparison is provided using data for the inception of sheet flow ( $c f$. Sec. 3.1 and Camenen \& Larson, 2006). When the sheet flow regime just appears, the bed is flat and the roughness height may be assumed to be equal to the grain-related roughness, i.e. $k_{s}=k_{s g}=2 d_{50}$. In Tab. 3, the "measured" (assuming $k_{s}=k_{s g}$ ) and "estimated" (based on the estimated ripple characteristics and using Eq. 17) roughness heights are compared.

The results from Tab. 3 clearly indicates the weakness of most of the predictive formulas. As they are based and fitted on experimental data where ripples exist, they do not forecast properly the absence of ripple. Only the Nielsen, Van Rijn and the Grasmeijer \& Kleinhans formulas predict a disappearance of the ripples even if some large uncertainties are encountered to predict this limit. The Nielsen formulas yield the best results just because it tends to underestimate $\theta_{c r, s f}$. 
Table 3: Prediction of the roughness height when the ripples are washed out and the sheet flow regime reached.

\begin{tabular}{l|cccc}
\hline Equation & $P 2$ & $P 5$ & $\mu_{f}$ & $\sigma_{f}$ \\
\hline Nielsen & $87 \%$ & $97 \%$ & +0.10 & 0.20 \\
Grant \& Madsen & $20 \%$ & $41 \%$ & +0.80 & 0.47 \\
Wikramanayake \& Madsen & $13 \%$ & $31 \%$ & +1.13 & 0.62 \\
Van Rijn & $41 \%$ & $57 \%$ & +0.56 & 0.52 \\
Mogridge et al. & $1 \%$ & $18 \%$ & +1.10 & 0.43 \\
Wiberg \& Harris & $0 \%$ & $0 \%$ & +1.68 & 0.39 \\
Grasmeijer \& Kleinhans & $24 \%$ & $78 \%$ & +0.51 & 0.26 \\
Soulsby \& Whitehouse & $0 \%$ & $0 \%$ & +1.71 & 0.33 \\
Eqs. 9 and 10, $\alpha_{c r}=0.01$ & $4 \%$ & $4 \%$ & +1.57 & 0.40 \\
Eqs. 9 and 10, $\alpha_{c r}=1$ & $47 \%$ & $55 \%$ & +0.63 & 0.65 \\
\hline
\end{tabular}

\section{A new model for the calculation of the ripple characteristics}

\subsection{Limits for the ripple existence}

A need clearly comes out from the above study for the estimation of the ripple characteristics, which is to estimate carefully their limits of existence, i.e. the critical Shields parameters corresponding to their appearance and disappearance. As concerns the appearance of ripples, it may be assumed that they exist as soon as sediments move. The critical Shields parameter for the inception of movement may be estimated using the equation proposed by Soulsby \& Whitehouse (1997),

$$
\theta_{c r}=\frac{0.30}{1+1.2 d_{*}}+0.055\left[1-\exp \left(-0.02 d_{*}\right)\right]
$$

where $d_{*}=\left[g(s-1) / \nu^{2}\right]^{1 / 3} d_{50}$ is the dimensionless grain size.

Camenen \& Larson (2006) proposed a maximum value of the wave orbital velocity $U_{w, c r, s f}$ for the inception of the sheet flow regime or wash-out of the wave ripples,

$$
U_{w, c r, s f}=8.35 \sqrt{(s-1) g \sqrt{d_{50} \delta_{w}}}\left(1+r_{w}\right)
$$

where $\delta_{w}=\sqrt{\nu T_{w} / \pi}$ is the thickness of the Stokes boundary layer and $r_{w}$ the wave asymmetry $\left(r_{w}=\left(u_{w, \max }-u_{w, \min } /\left(u_{w, \max }+u_{w, \min }\right)\right.\right.$ with $u_{w, \max }$ and $u_{w, \min }$ are the maximum onshore and minimum offshore velocity, respectively). A simple empirical equation has been suggested by Hanson \& Camenen (2007) for the wave induced friction factor (validated with plane bed data), which may be written as follows:

$$
f_{w}=\frac{0.5}{\sqrt{\pi}} d_{*}{ }^{-3 / 4} \frac{d_{50}}{\delta_{w}}
$$


Thus, an expression for the critical Shields parameter $\theta_{c r, s f}$ for the inception of sheet-flow may be obtained from Eqs. 1, 6] and 7.

$$
\theta_{c r, s f}=10 d_{*}{ }^{-3 / 4} \sqrt{\frac{d_{50}}{\delta_{w}}}\left(1+r_{w}\right)^{2}
$$

Using the same experimental data as Camenen \& Larson (2006), Eq. 8 shows relatively good results ( $c f$. Fig. 1). It can also be observed from the data that the critical Shields parameter for the inception of sheet flow may vary from 0.2 to 2.0.

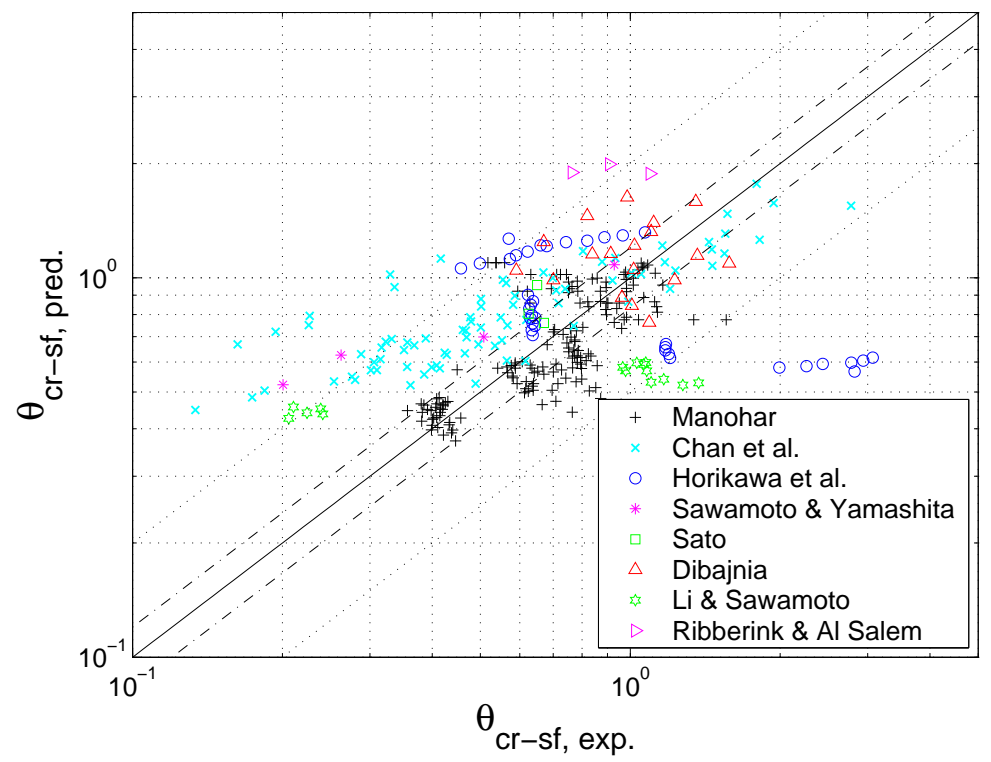

Figure 1: Estimation of the critical Shields parameter for the inception of waverelated sheet flow using Eq. 8,

It should be noted that the threshold of sheet-flow in term of $\left(A_{w} / d_{50}\right)_{c r, s f}$ (as used by Wiberg \& Harris, 1994, or Soulsby \& Whitehouse, 2005) varies from 60 to 6000 using the same experimental data as Camenen \& Larson (2006). Thus, the Wiberg \& Harris and Soulsby \& Whitehouse formulas seem to overestimate this threshold as they assume $\left(A_{w} / d_{50}\right)_{c r, s f} \approx 6000$.

\subsection{New equations for the description of the ripple charac- teristics}

Following the previous observations, two simple equations function of the skin Shields parameter and its critical values only (inception of movement and inception of sheet flow) are proposed to predict the ripple length and steepness, respectively:

$$
\begin{aligned}
\frac{L_{r}}{A_{w}} & =\left(\frac{L_{r}}{A_{w}}\right)_{e q} f\left(\frac{\theta_{c r}}{\theta_{w g}}\right) f\left(\frac{\theta_{w g}}{\theta_{c r, s f}}\right) \\
\frac{H_{r}}{L_{r}} & =\left(\frac{H_{r}}{L_{r}}\right)_{e q} f\left(\frac{\theta_{c r}}{\theta_{w g}}\right) f\left(\frac{\theta_{w g}}{\theta_{c r, s f}}\right)
\end{aligned}
$$


where the subscript $e q$ corresponds to the equilibrium value of the ratio $L_{r} / A_{w}$ and $H_{r} / L_{r}$, respectively, $\theta_{w g}$ is the grain-related Shields parameter (computed assuming $\left.k_{s}=2 d_{50}\right)$ and $f$ is a function defining the existence of the ripples or not:

$$
f(x)=\exp \left(-\alpha_{c r} x^{4}\right)
$$

with $\alpha_{c r}$ a coefficient.

Grant \& Madsen (1982), Van Rijn (1993) or Grasmeijer \& Kleinhans (2004) observed that $\left(L_{r} / A_{w}\right)_{e q}$ is a decreasing function of $\Psi_{w}$ or $\theta_{w}$. On the other hand, Mogridge et al. (1994) found a relationship between the ratio $L_{r} / d_{50}$ and the wave period parameter $\chi$. In Fig. 2, the ripple wavelength normalised by the wave orbital semi-excursion $L_{r} / A_{w}$ was plotted against the grain-related Shields parameter $\theta_{w g}$ with the wave period parameter $\chi$ emphasised (for practical purpose, flat beds are represented in this figure by the value $L_{r} / A_{w}=10^{-2}$ ). It appears that the ratio $L_{r} / A_{w}$ does decrease with the grain-related Shields parameter $\theta_{w g}$ but with a varying slope that depends on $\chi$. A relationship for the equilibrium value of the ratio $L_{r} / A_{w}$ is suggested as a function of $\theta_{w g}$ and $\chi$ :

$$
\left(\frac{L_{r}}{A_{w}}\right)_{e q}=1.6 \exp \left(-5 \times 10^{-3} \chi^{-0.4}\right) \theta_{w g}^{-0.025 \chi^{-0.2}}
$$

Some uncertainties obviously exist in the determination of the critical Shields parameter for the inception of sheet flow. Indeed, many data from Hanes et al. indicate a disappearance of ripples for much smaller Shields parameter than expected. Unlike the observations by Wiberg \& Harris (1994), the collected data do not show any clear relationship between the ripple length and the grain size (when $A_{w}>3000$ ) corresponding to the anorbital ripples. Only orbital and suborbital ripples were observed. It should also be noted that there is a relationship between $\chi$ and Eq. 8 for $\theta_{c r, s f}$, and $\chi=10^{-8} \pi^{-2} \theta_{c r, s f}{ }^{8} d_{*}^{3}$.

The ripple steepness was often observed to be nearly constant, and $H_{r} / L_{r} \approx 0.16$ (Wiberg \& Harris, 1994). As observed by Soulsby \& Whitehouse (2005), the ripple steepness appears to be strongly dependent on the ratio $A_{w} / d_{50}$ with a smaller steepness for suborbital ripples $\left(A_{w} / d_{50}>1000\right)$. In Fig. 3, the ripple steepness $H_{r} / L_{r}$ was plotted against wave orbital semi-excursion normalised by the median grain size $A_{w} / d_{50}$ with the wave period parameter emphasised. A clear decrease of the ripple steepness with the ratio $A_{w} / d_{50}$ may be observed. The following equation for the equilibrium ripple steepness is then suggested,

$$
\left(\frac{H_{r}}{L_{r}}\right)_{e q}=0.16 \exp \left[-1.0 \times 10^{-7}\left(\frac{A_{w}}{d_{50}}\right)^{2}\right]
$$

The introduction of the function $f$ in Eqs. 9 and 10 was proposed to properly evaluate the non-existence of the ripples. As some uncertainties exist on the prediction of $\theta_{c r}$ or $\theta_{c r, s f}$, this exponential function allows positive values for $H_{r}$ and $L_{r}$, but much smaller than their equilibrium values.

In Fig. 4, Eqs. 9 and 10 are plotted versus the grain-related Shields parameter for four values of the critical Shields parameter for the inception of sheet flow 


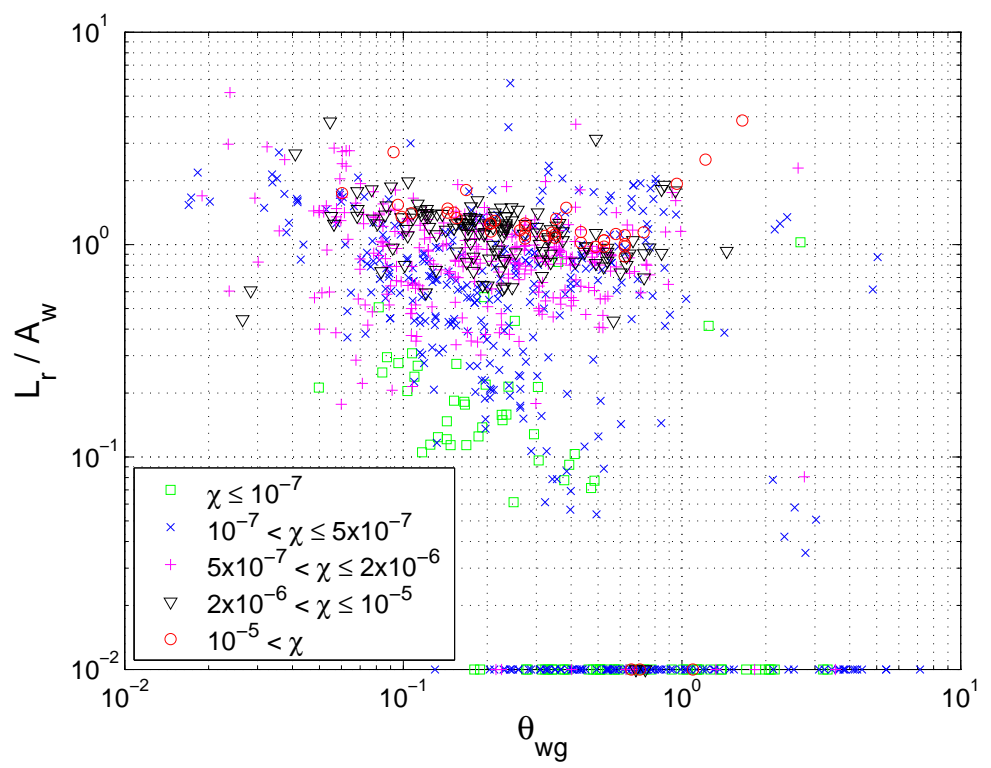

Figure 2: Wave-generated ripple wavelength normalised by the wave orbital semiexcursion as a function of the grain-related Shields parameter with the wave period parameter emphasised.

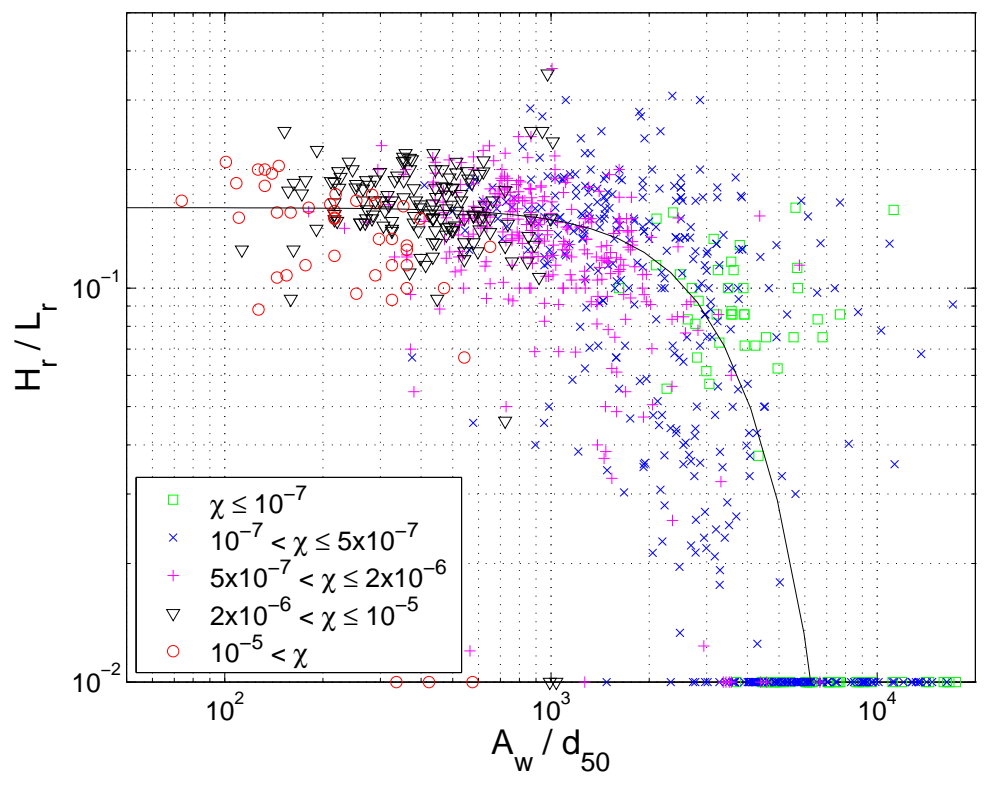

Figure 3: Wave-generated ripple steepness as a function of the wave orbital semiexcursion normalised by the median grain size with the wave period parameter emphasised (the solid line corresponds to Eq. 13).

$\left(\theta_{c r, s f}=0.75,1.0,1.25\right.$ and 1.5$)$ for a given grain size $\left(d_{50}=0.3 \mathrm{~mm}\right)$. The wave period is estimated using the relationship between $\chi$ and Eq. 8 for $\theta_{c r, s f}$. As expected, the proposed formulas yield a ripple steepness which depends mainly on the ratio $\theta_{w g} / \theta_{c r, s f}$ and a slightly more complex function for the ratio $H_{r} / A_{w}$. Compar- 
ing with the experimental data ( $c f$. Fig. 2), it appears that Eqs. 9, 10, 11 (with $\alpha_{c r}=1$ ), 12, and 13, provide a good overview of the experimental data as soon as the critical Shields parameter for the inception of sheet flow is properly estimated. When $\theta_{c r, s f}<0.8$ ( or $\chi<10^{-7}$ ), ripples present much smaller height and steepness or even may not occur whatever the Shields parameter.

(a)

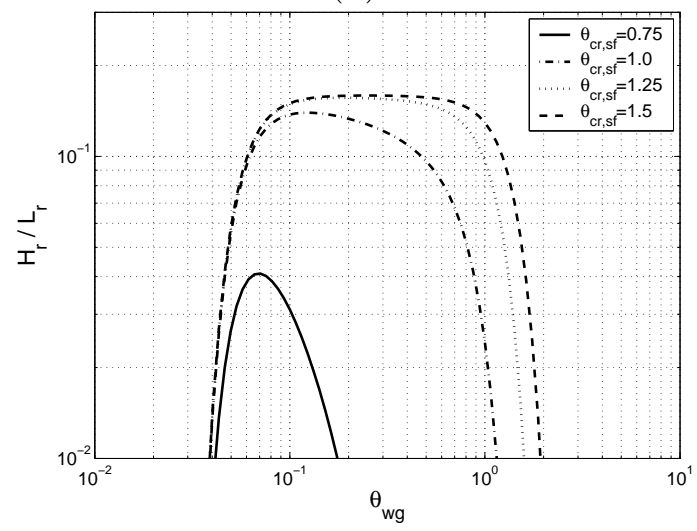

(b)

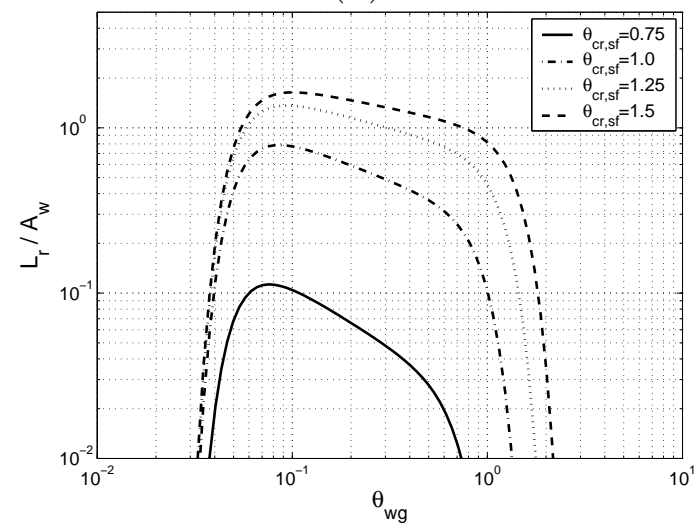

Figure 4: Ripple steepness $H_{r} / L_{r}$ (a) and ratio $L_{r} / A_{w}$ (b) versus the skin Shields parameter for several values of the critical Shields parameter for the inception of sheet flow $\theta_{c r, s f}$ using Eqs. 9 and [10 with $\alpha_{c r}=1$ and $d_{50}=0.3 \mathrm{~mm}$.

Using Eqs. 5 and 8 for the estimation of the different critical Shields parameter, Eqs. 9 and 10 yield good results for the prediction of the ripple characteristics (see Tab. 2). Of course, even if a large data set has been used, these good results (compared to the other formulas) are slightly biased as the newly proposed formulas were optimized using this data set. As shown in Tab. 3, the correct prediction of $\theta_{c r, s f}$ and its integration in the ripple formulas are fundamental not to overestimate largely the roughness (and thus the Shields parameter) when the ripples are washed out. The influence of the coefficient $\alpha_{c r}$ in Eq. 11 appears to be significant when sheet flow regime is reached. Indeed, if the choice of $\alpha_{c r}=0.01$ or 1 does not affect significantly the results for the prediction of the ripple characteristics (results slightly poorer when $\alpha_{c r}=1$, see Tab. 2), the choice of $\alpha_{c r}=1$ seems much more appropriate to predict the absence of ripples ( $c f$. Tab. 33).

\subsection{Large wave ripples}

For large wave ripples (LWR), all the proposed formulas overestimate the ripple height and steepness. In Fig. 5, the wavelength and steepness with LWR data from Hume et al. (1999) and Hanes et al. (2001) have been plotted against the grain-related Shields parameter. Compared to Eqs. 9 and 10, the behaviour against $\theta_{w g}$ seems to be properly reproduced (decrease of $L_{r} / A_{w}$ with an increasing $\theta_{w g}$ ). However, the ripple wavelength is underestimated by a factor 4 to 8. Apart from the data by Hume et al., the wave steepness is also underestimated by a factor 4 to 8 , which means that the ripple height is properly estimated. Thus, some LWR 
could correspond to a more complex energetic system at the boundary layer where several frequencies coexist due to the frequency-dependent reduction of fluid orbital amplitude with depth. A higher frequency may control the ripple height whereas the smaller frequency may control the ripple length. Another hypothesis for the occurrence of the LWR is the relict (or fossil) ripples built during a previous event that may affect the ripple characteristics during the following period.
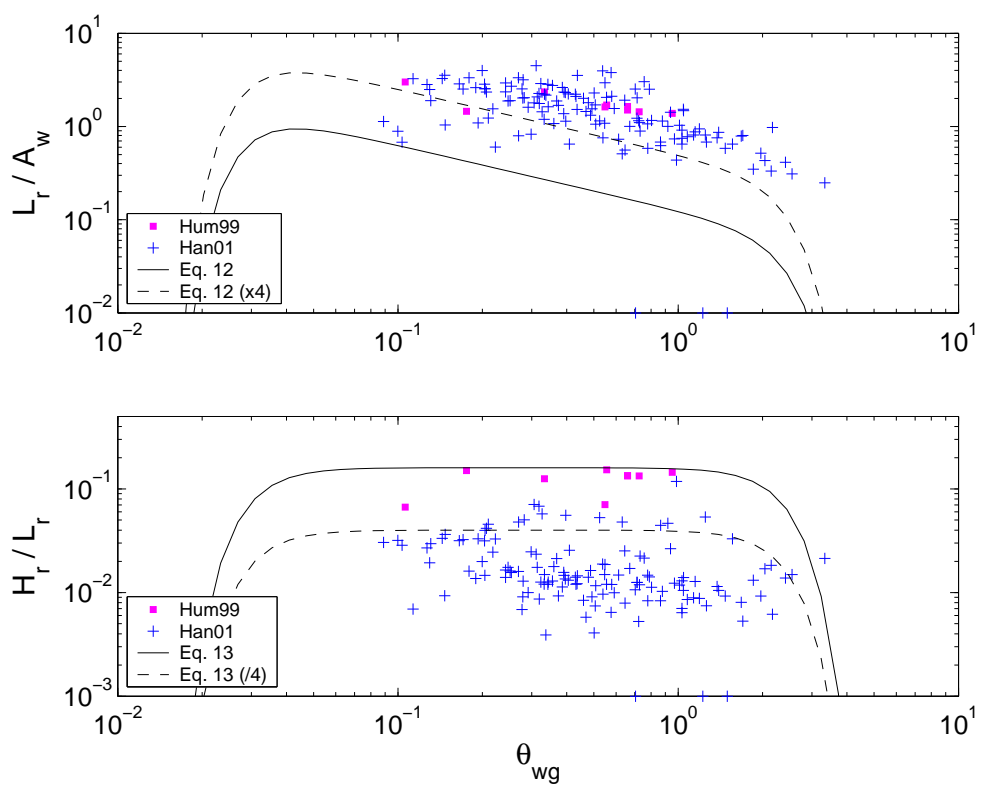

Figure 5: Large wave ripple (LWR) wavelength (normalised by the wave orbital semi-excursion) and steepness as a function of the grain-related Shields parameter.

\subsection{Effects of a steady current}

When a current was superimposed on the waves, Van Rijn et al. (1993) observed that ripples become asymmetrical and rapidly three-dimensional with increasing current. Khelifa \& Ouellet (2000) showed that positive and negative longitudinal current velocities produce a similar effect on the ripple characteristics, i.e. an increase of the ripple length and height with increasing current. Based on kinetic considerations, they suggested to use the effective fluid orbital semi-excursion instead of the wave orbital semi-excursion,

$$
\begin{aligned}
A_{c w} & =\sqrt{A_{w}{ }^{2}+\left(\frac{T_{w} U_{c}}{2}\right)^{2}+A_{w} T_{w}\left|U_{c}\right||\cos \phi|} \\
& =A_{w} \sqrt{1+\left(\frac{\pi U_{c}}{U_{w}}\right)^{2}+2 \pi \frac{\left|U_{c}\right|}{U_{w}}|\cos \phi|}
\end{aligned}
$$

where $U_{c}$ is the steady current and $\phi$ the angle between the wave and current directions. Following this idea, the Shields parameter in a wave and current interaction 
may be estimated such as $\theta_{c w}=\theta_{w}\left(A_{c w} / A_{w}\right)^{2}$. It is thus possible to use Eqs. 9 to 13 in case of a wave and current interaction by replacing $A_{w}$ and $\theta_{w}$ by $A_{c w}$ and $\theta_{c w}$, respectively.

In Fig. 6, the ratio between the predicted (using Eqs. 9 to 13) and measured ripple length and height is plotted versus the ratio $\left|U_{c}\right| / U_{w}$. It appears that, if both ripple length an height are properly estimated when $U_{c}=0$, a significant over-estimation exists as soon as $\left|U_{c}\right| / U_{w}>10^{-2}$ (where $L_{r, \text { pred }} / L_{r, \text { meas }} \approx 2$ ). No explanation was found to explain this reduction in the ripple dimensions due to a relatively low current. The change from $2 \mathrm{D}$ symmetrical ripples to $3 \mathrm{D}$ asymmetrical ripples may affect the average ripple length and height. When $\left|U_{c}\right| / U_{w}>10^{-2}$, ripple characteristics are increasing as predicted by Eq. 14. As observed by Van Rijn \& Havinga (1995), the angle between the wave and current directions has no significant influence on the ripple dimensions.

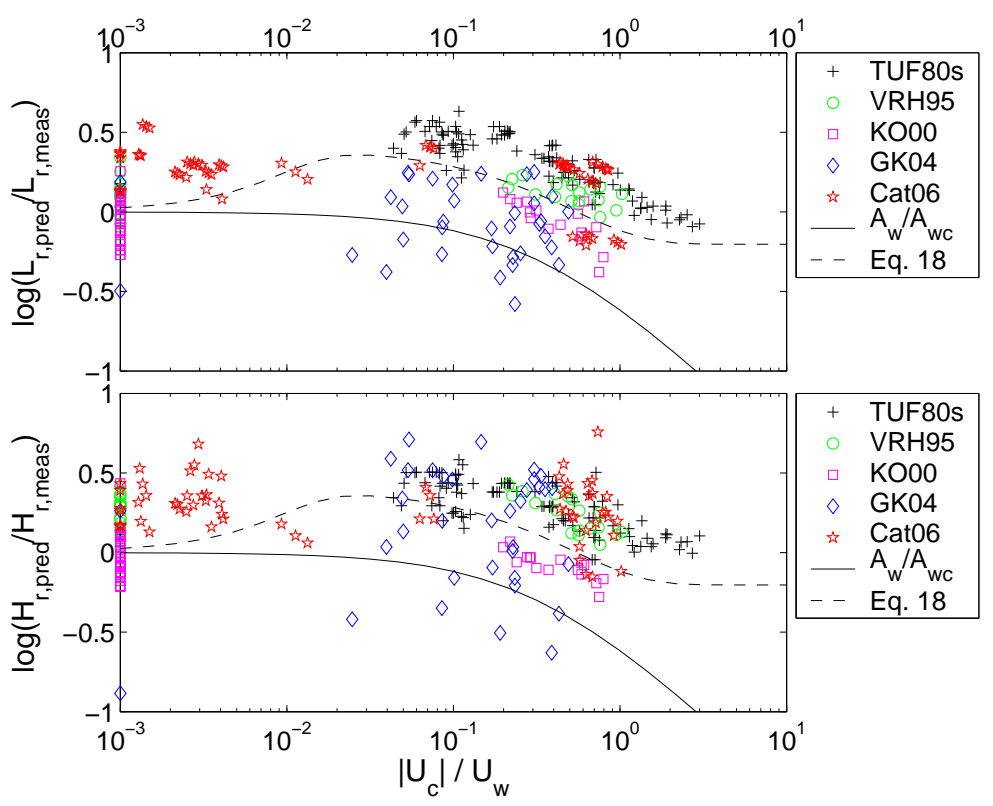

Figure 6: Prediction of the ripple wavelength and steepness using Eqs. 9] and 10 as a function of the ratio between the steady current amplitude of the wave orbital velocity.

As the steady current appears to induce two opposite effects on the ripple dimensions (decreasing when $\left|U_{c}\right| / U_{w}<3 \times 10^{-2}$ and increasing $\left|U_{c}\right| / U_{w}>3 \times 10^{-2}$ ), Eqs. 9] to 13 for waves alone still yield correct results ( $c f$. Tab. 4). Using Eq. 14 improves the behaviour of the prediction against the ratio $\left|U_{c}\right| / U_{w}$. However, it tends to overestimate ripple dimensions; and the critical Shields parameter for inception of sheet flow is reached more easily (as $\theta_{c w}>\theta_{w}$ ), which yields a too fast disappearance of the ripples. An empirical coefficient for Eqs. 9 and 10 is suggested to improve the prediction of the ripple characteristics under waves and current:

$$
r_{w c}=\left[1-0.6 \tanh \left(50 \frac{\left|U_{c}\right|}{U_{w}}\right)\right]\left[1+3 \tanh \left(\frac{\left|U_{c}\right|}{U_{w}}\right)\right]
$$


As observed in Tab. 4, Eq. 15 significantly improves the results for the ripple prediction in case of a wave and current interaction.

Table 4: Prediction of the ripple characteristic under waves and current.

\begin{tabular}{l|cccc|cccc}
\hline & \multicolumn{4}{|c|}{ ripple length $L_{r}$} & \multicolumn{4}{c}{ ripple height $H_{r}$} \\
Equation & $P 2$ & $P 5$ & $\mu_{f}$ & $\sigma_{f}$ & $P 2$ & $P 5$ & $\mu_{f}$ & $\sigma_{f}$ \\
\hline Eqs. 9 to 13 & $69 \%$ & $100 \%$ & +0.16 & 0.24 & $59 \%$ & $98 \%$ & +0.21 & 0.24 \\
Eqs. 9 to 13 using Eq. 14 & $45 \%$ & $95 \%$ & +0.16 & 0.52 & $50 \%$ & $91 \%$ & -0.08 & 1.15 \\
Eqs. 9 to 13 using Eq. 15] & $81 \%$ & $99 \%$ & +0.08 & 0.23 & $73 \%$ & $98 \%$ & +0.14 & 0.24 \\
\hline
\end{tabular}

\section{Estimation of the wave-related Shields para- meter in case of ripples}

The calculation of the bed shear stress in the nearshore is not trivial. The main difficulty lies in the determination of the wave-related friction factor $f_{w}$. $f_{w}$ was calculated using the Swart formula (1974):

$$
f_{w}=\min \left\{\exp \left[-6.0+5.2\left(\frac{A_{w}}{k_{s}}\right)^{-0.19}\right], 0.3\right\}
$$

where the roughness height $k_{s}$ needs to be estimated. Many uncertainties are then combined: they stem from the prediction of ripple characteristics but also their effects on the bed shear stress and the limits for the presence of ripples.

\subsection{Calculation of the roughness height for a rippled bed}

In the off-shore region where the ripples are generally observed, the shear stress due to the current is often much smaller than the shear stress due to the waves, and then, may be neglected in this study. The total roughness height $k_{s}$ was estimated using the method proposed by Soulsby (1997) by summing the grain-related $k_{s g} \approx 2 d_{50}$, form-drag $k_{s f}$, and sediment transport $k_{s s}$ components, respectively: $k_{s}=k_{s g}+$ $k_{s f}+k_{s s}$. When sheet flow regime occurs, the Wilson (1989) formula was used, i.e. $k_{s s}=5 \theta_{w} d_{50}$. Thereafter, the grain related Shields parameter $\theta_{w g}$ based on $k_{s g}$ will be distinguished from the total Shields parameter $\theta_{w}$ based on $k_{s}$.

The ripple-related roughness height $k_{s f}$ is generally calculated using the following formula,

$$
k_{s f}=a_{r} \frac{H_{r}^{2}}{L_{r}}
$$

where $a_{r}$ is a constant $\left(5<a_{r}<40\right)$. Nielsen (1992) suggested $a_{r}=8$ whereas Van Rijn (1993) proposed $a_{r}=20$. 
More recently, Kim (2004) investigated the effective form roughness of the wave ripples using a numerical model with a mixing length hypothesis to simulate the wave boundary layer flow over ripples. He obtained the ripple-length average bed shear stress (or form drag friction) from integration of the computed pressure field. An interesting conclusion from his study is that the effective roughness for sharpcrested ripples becomes about three times larger than that for the sinusoidal shape. It means that the presence of a steady current may considerably modify (reduce) the effective roughness as it will smooth the ripple crests. Nevertheless, this effect will be neglected in this study but may partly explain the different values suggested for $a_{r}$. Another explanation is the choice of the formula for $f_{w}$, which may influence afterwards the choice for $a_{r}$.

The ratio $H_{r}^{2} / L_{r}$ appeared to be a fundamental parameter for the estimation of the roughness height in the ripple regime, and thus for the estimation of the total Shields parameter. In Fig. 7(a), the ratio $H_{r}{ }^{2} / L_{r}$ is plotted versus the ratio $A_{w} / d_{50}$ using all the different studied formulas (assigned with initials of the authors or with the three first letters of the first author): The Nielsen (1981, Nie), Grant \& Madsen (1982, GM), Wikramanayake \& Madsen (1991, WM), Van Rijn (1993, VR), Mogridge et al. (1994, Mog), Wiberg \& Harris (1994, WH), Grasmeijer \& Kleinhans (2004, GK), Soulsby \& Whitehouse (2005, SW), and Williams et al. (2005, Wil) formulas. As most beaches consist of sand with a grain size ranging from $d_{50}=0.15$ to $0.35 \mathrm{~mm}$, and because the wave period does not vary on a cross-shore profile (if the energy transfer to nearly harmonic wave components is neglected), this sensitivity analysis is presented for a varying wave excursion only, assuming a constant median grain size $d_{50}=0.25 \mathrm{~mm}$ and a constant wave period $T_{w}=6 \mathrm{~s}$. It should be noted that the calculated values for $H_{r}{ }^{2} / L_{r}$ do vary significantly depending on the fixed parameters $\left(d_{50}\right.$ or $\left.T_{w}\right)$. For the Soulsby \& Whitehouse formula, $U_{1 / 10}$ was used instead of $U_{1 / 3}$ to represent the wave orbital velocity in case of irregular waves. When applied to regular waves, the curve should then be shifted rightward relative to the other curves.

Even if large discrepancies are observed between the different formulas, a similar trend for the coefficient $H_{r}{ }^{2} / L_{r}$ may be observed:

- for $A_{w} / d_{50}<1000$ (orbital ripples), $H_{r}{ }^{2} / L_{r}$ is an increasing function of $A_{w} / d_{50}$.

- when $1000<A_{w} / d_{50}<3000$ (suborbital ripples), $H_{r}{ }^{2} / L_{r}$ reaches a maximum which is similar whatever the formula, and then decreases. $\left(H_{r}{ }^{2} / L_{r}\right)_{e q} \approx$ $6 \times 10^{-3}$. This value appeared to be an increasing function of $d_{50}$ or $T_{w}$.

- for $A_{w} / d_{50}>3000$ (anorbital ripples), $H_{r}{ }^{2} / L_{r}$ decreases rapidly toward zero.

It is also in accordance with Van Rijn (2007), who suggested a constant value for the ripple induced roughness height $k_{s f}=150 d_{50}$ when $\Psi_{w}<50$ with a quick decrease afterwards. Then, the main differences observed between the formulas are not in the magnitude of the coefficient $H_{r}{ }^{2} / L_{r}$ but in the limits for the ripple presence (critical Shields parameters for the inception of movement and inception of sheetflow, respectively). 
(a)

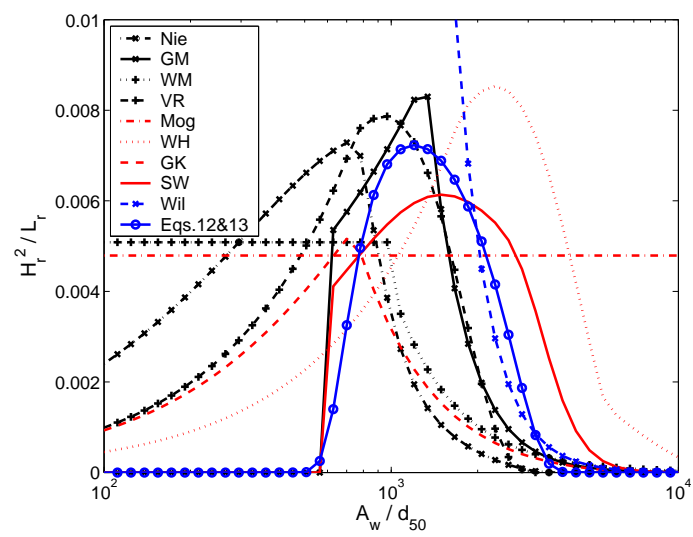

(b)

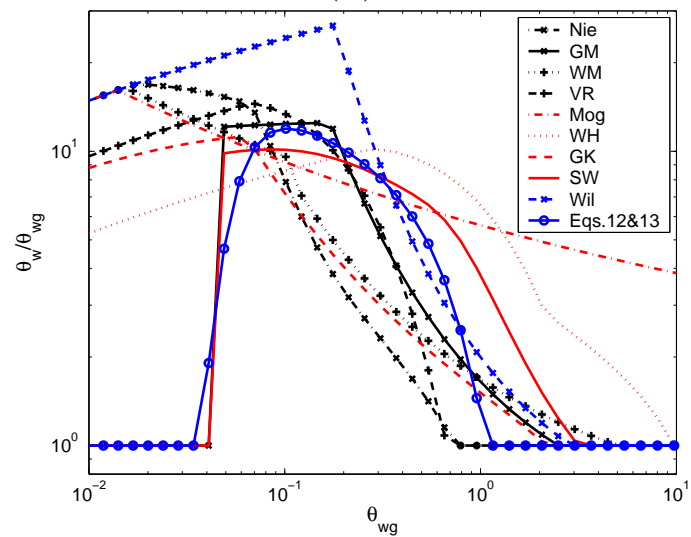

Figure 7: Ratio $H_{r}{ }^{2} / L_{r}$ versus the ratio $A_{w} / d_{50}$ (a) and the induced Shields parameter $\theta_{w}$ versus the ratio $A_{w} / d_{50}$ (b) for a constant medium grain size $d_{50}=0.25 \mathrm{~mm}$ and a constant wave period $T_{w}=6 \mathrm{~s}$ using the different studied formulas for the ripple characteristics (the roughness height $k_{s}$ was calculated using Eq. 17 with $\left.a_{r}=10\right)$.

\subsection{Influence of the ripple characteristics on the total Shields parameter}

Using Eq. 17 with $a_{r}=10$ for the calculation of the ripple-induced roughness height for the eight studied formulas, the total shear stress was estimated for the same conditions as in Fig. 7(a); i.e. with fixed median grain size and wave period. This condition is quite coherent with what is observed on a beach for a specific time: a large range of bed shear stresses with nearly constant median grain size and wave period. Fig. 7(b) shows a typical evolution of the ratio between the total and skin wave-related Shields parameters as a function of the skin wave-related Shields parameter using the different studied formulas.

For very small skin shear stress $\left(\theta_{w g}<\theta_{c r}\right)$, as only the "GM" and "SW" formulas takes into account $\theta_{c r}$, only these formulas yield realistic results. The other formulas predict a Shields parameter one order of magnitude larger than the skin Shields parameter. Even if there are some uncertainties in the prediction of the critical Shields parameter for the inception of movement ( $c f$. Eq. 5 ), it is necessary to take into account this critical value.

For relatively small skin shear stresses $\left(\theta_{c r}<\theta_{w g}<0.2\right)$, most of the formulas present similar results which indicate a total Shields parameter approximately 5 to 10 times larger than the grain-related Shields parameter. It is possible to define an equilibrium value for the ratio between the total and the skin-related Shields parameters $\left(\theta_{w} / \theta_{w g}\right)_{e q}$.

Then, for larger skin shear stress $\left(0.2<\theta_{w g}<\theta_{c r, s f}\right)$, the ratio $\theta_{w} / \theta_{w g}$ is decreasing with an increasing skin-related Shields parameter. The ratio also appeared to be a decreasing function of $d_{50}$ or $T_{w}$ (suborbital regime). The uncertainties in the prediction of the ripples wash-out are very large. Even if Wiberg \& Harris (1994) 
observed that ripples cannot exist for $A_{w} / d_{50}>6000$, most of the formulas, including the "WH" formula, yield a wash-out of the ripples $\left(\theta_{w}=\theta_{w g}\right)$ for larger values of $A_{w} / d_{50}$. As no limit is provided, the "Mog" equation yields a presence of ripples whatever the shear stress, which is unrealistic. On the contrary, the "Nie" and "VR" formulas yield a wash-out of the ripples for $\theta_{w g} \approx 0.7-0.9$. This is more in agreement with the observations by Wiberg \& Harris even if bed forms have been observed for higher shear stresses. An important parameter for the estimation the roughness height due to ripple is therefore the critical Shields parameter for the inception of sheet flow. It may also be observed that these formulas induce a nearly constant (even decreasing for the "VR" formula) total Shields parameter for an increasing wave orbital velocity when suborbital ripples are present $\left(1000<A_{w} / d_{50}<5000\right)$.

It should be noted that $f_{w}$ and then $\left(\theta_{w} / \theta_{w g}\right)_{e q}$ are functions of the coefficient $a_{r}$ (cf. Eq. 17) and strongly depend on the chosen value for $a_{r}$. Based on Kim (2004) numerical results, very different roughnesses are observed depending on the shape of the ripple. For sharp-crested ripples (pure oscillatory flow), $a_{r}=13.4$ is obtained whereas using a sinusoidal profile with the same characteristics, $a_{r} \approx 6.3$ is obtained. In case of a wave and current interaction, where the shape of the ripples is much smoother, a relatively low coefficient should thus be used.

Soulsby \& Whitehouse (2007?) proposed an alternative approach to compute the friction coefficient $f_{w}$ using the drag coefficient approach on strip roughness elements :

$$
f_{w}=\frac{M_{c d}}{A_{w}} \frac{H_{r}{ }^{2}}{L_{r}}
$$

with $M_{c d}$ a coefficient (Soulsby \& Whitehouse suggested to used $M_{c d}=5$ ). In Fig. 8 , the friction coefficient is plotted versus the ratio $\left(H_{r}{ }^{2} / L_{r}\right) / A_{w}$ with observed values from experiments of Jonsson \& Carlsen (1976), and Mathisen \& Madsen (1996) and numerical model results of Andersen (2001) and Kim (2004). Eq. 16 with Eq. 17 and Eq. 18 yield similar results when $f_{w}>0.05$ and $M_{c d} \approx a_{r} / 2$. From the observed values, it may be noted that $a_{r}\left(M_{c d}\right)$ may vary from 5 to 30 (2.5 to 15). In the same way, $\left(\theta_{w} / \theta_{w g}\right)_{e q}$ may vary by a factor 6 depending on the choice of $a_{r}$ (or $M_{c d}$ ).

\subsection{Estimation of the total Shields parameter directly from the skin Shields parameter}

Following the observations in section 4.2 (and the trends of the empirical formulas in Fig. 7, as well as the study of ripple characteristics proposed in section 3.2) and using $\theta_{c r}$ (Eq. 51) and $\theta_{c r, s f}$ (Eq. 8), a simple formula is proposed to estimate the total Shields parameter due to the ripples directly from the grain-related Shields parameter:

$$
\frac{\theta_{w}}{\theta_{w g}}=1+\left[\left(\frac{\theta_{w}}{\theta_{w g}}\right)_{e q}-1\right] f_{2}\left(\frac{\theta_{c r}}{\theta_{w g}}\right) f_{2}\left(\frac{\theta_{w g}}{\theta_{c r, s f}}\right)
$$




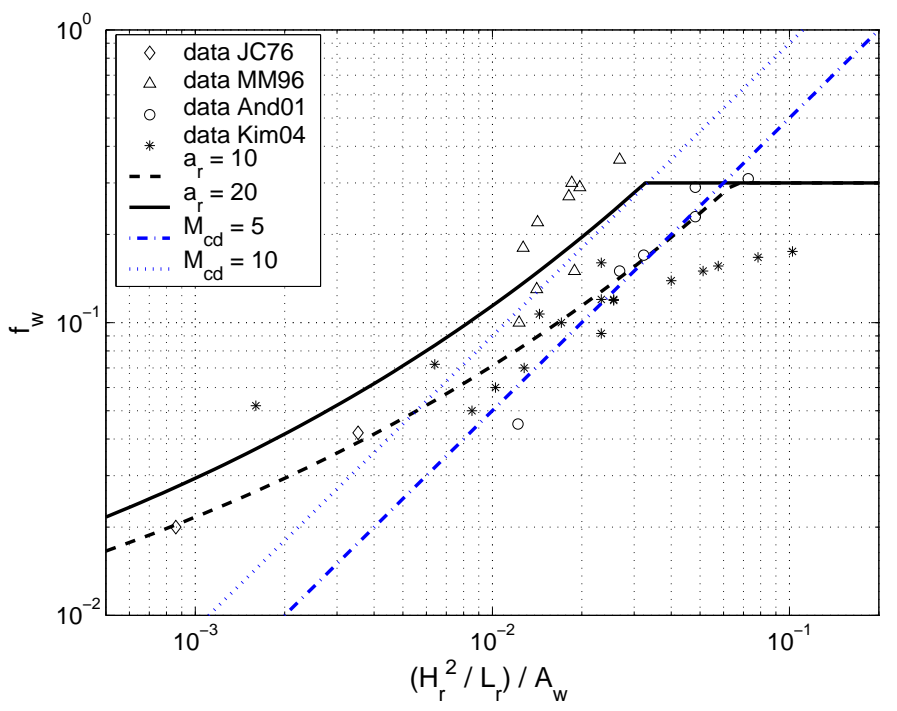

Figure 8: Wave related friction coefficient $f_{w}$ as a function of the ratio $\left(H_{r}{ }^{2} / L_{r}\right) / A_{w}$ (Eq. 16 and Eq. 18 are plotted, using Eq. 17 with $a_{r}=10$ and 20, and $M_{c d}=5$ and 10, respectively, together with experimental data from Jonsson \& Carlsen, 1976, and Mathisen \& Madsen, 1996, and numerical data from Andersen, 2001, and Kim, 2004).

where the function $f_{2}$ was found to be $f_{2}(x)=f^{2}(x)=\exp \left(-2 \alpha_{c r} x^{4}\right)$, and an estimation of the equilibrium value of the ratio $\theta_{w} / \theta_{w g}$ may be:

$$
\left(\frac{\theta_{w}}{\theta_{w g}}\right)_{e q}=1+1.0 a_{r} \exp \left(-1.5 \times 10^{-6} \frac{\theta_{w g}}{\chi}\right)
$$

Using Eqs. 17 (with $a_{r}=10$ ), 5, 8, 9 and 10, it is possible to estimate the total Shields parameter as a function of the skin Shields parameter. Fig. 9 presents a comparison between the method based on the ripple characteristics (1) and the empirical equation directly based on the skin Shields parameter (2: Eqs. 19] and 201). One can observe that both methods present very similar results. A truncation may appear using the first method for large grain size or wave period values combine with a large $a_{r}$-value as $f_{w}$ reaches its maximum $\left(\max \left(f_{w}\right)=0.3\right.$, cf. Eq. 16). For fine sediments or short wave periods, the behaviour is similar to the Van Rijn formula, i.e. for $0.5<\theta_{w g}<1$ (cf. Fig. [7(b)), the total Shields parameter is a fast decreasing function of the grain-related Shields parameter, thus a decreasing function of the wave height, which needs to be validated. For coarser sediments or longer wave periods (i.e. for larger values of $\theta_{c r, s f}$ ), this effect is not as strong.

\subsection{Comparison with data}

Tab. 5 presents the statistical results on the prediction of the total Shields parameter using the different formulas for the prediction of ripple characteristics. As for the previous calculations, Eq. 17 with $a_{r}=10$ was used to estimate the rippleinduced roughness height for both measured and estimated data. It appeared logically that the formulas which predict the best ripple characteristics yield the most 
(a)

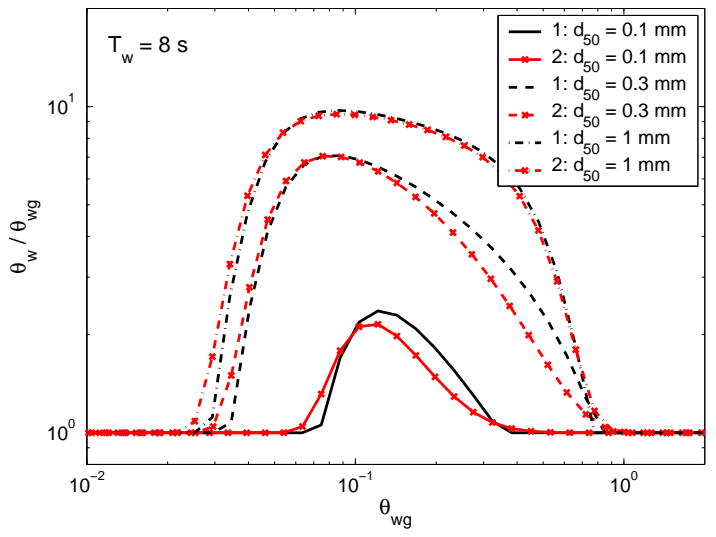

(b)

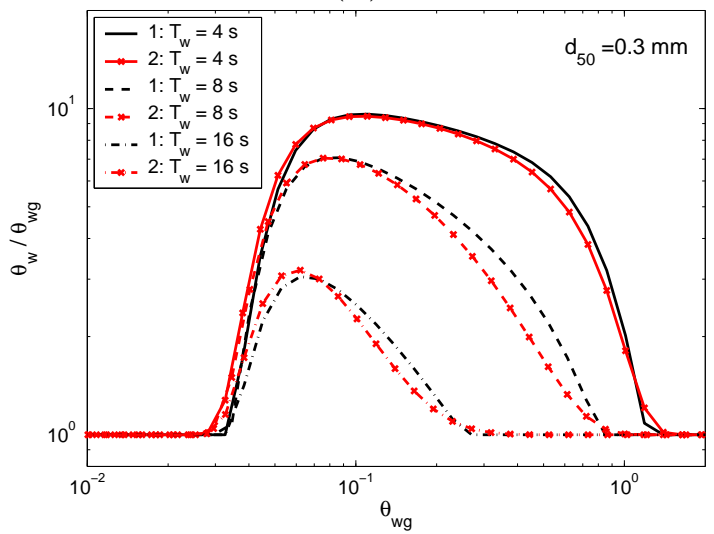

Figure 9: Total Shields parameter versus the skin Shields for several median grain sizes (a) and wave periods (b) using the classical method based on the prediction of the ripple characteristics (1: black lines) or Eq. 19 (2: red lines with crosses).

accurate predictions of the total Shields parameter. Thereby, the Van Rijn, Soulsby \& Whitehouse formulas as well as the suggested formula (Eqs. 9] and 10) yield the best results. Using $\alpha_{c r}=1$ does influence the results as the Shields parameter is strongly underestimated when $\theta_{w g}<\theta_{c r}$ whereas ripples were observed. In the same way, Eq. 19, which estimated the total Shields parameter directly from the skin Shields parameter, yields satisfactory results although it may underestimate results for $\theta_{w g}<\theta_{c r}$ or $\theta_{w g}>\theta_{c r, s f}$. On the other hand, much better results are observed for the data set where ripples are washed out.

Table 5: Prediction of the Shields parameter from the estimation of the ripple characteristics using various empirical equations and the collected experimental data.

\begin{tabular}{l|cccc|cccc}
\hline & \multicolumn{4}{|c|}{ ripple data } & \multicolumn{4}{|c}{ data for $\theta_{w}=\theta_{c r, s f}$} \\
Equation & $P 1.2$ & $P 2$ & $\mu_{f}$ & $\sigma_{f}$ & $P 1.2$ & $P 2$ & $\mu_{f}$ & $\sigma_{f}$ \\
\hline Nielsen & $19 \%$ & $48 \%$ & -0.24 & 0.42 & $85 \%$ & $96 \%$ & +0.04 & 0.10 \\
Grant \& Madsen & $16 \%$ & $53 \%$ & -0.02 & 0.45 & $17 \%$ & $46 \%$ & +0.36 & 0.26 \\
Wikramanayake \& Madsen & $15 \%$ & $52 \%$ & -0.14 & 0.40 & $10 \%$ & $38 \%$ & +0.58 & 0.38 \\
Van Rijn & $29 \%$ & $64 \%$ & +0.005 & 0.40 & $39 \%$ & $59 \%$ & +0.27 & 0.28 \\
Mogridge et al. & $13 \%$ & $43 \%$ & +0.51 & 0.48 & $0 \%$ & $14 \%$ & +0.47 & 0.16 \\
Wiberg \& Harris & $18 \%$ & $62 \%$ & +0.09 & 0.35 & $0 \%$ & $0 \%$ & +0.83 & 0.14 \\
Grasmeijer \& Kleinhans & $10 \%$ & $50 \%$ & -0.15 & 0.39 & $15 \%$ & $82 \%$ & +0.20 & 0.12 \\
Soulsby \& Whitehouse & $25 \%$ & $73 \%$ & +0.02 & 0.33 & $0 \%$ & $0 \%$ & +0.85 & 0.11 \\
Eqs. 9] and 10, $\alpha_{c r}=0.01$ & $36 \%$ & $78 \%$ & -0.05 & 0.30 & $4 \%$ & $5 \%$ & +0.79 & 0.22 \\
Eqs. 9] and 10, $\alpha_{c r}=1$ & $34 \%$ & $74 \%$ & -0.11 & 0.33 & $46 \%$ & $58 \%$ & +0.29 & 0.31 \\
Eq. 19] & $32 \%$ & $71 \%$ & -0.12 & 0.36 & $30 \%$ & $45 \%$ & +0.41 & 0.36 \\
\hline
\end{tabular}

In Fig. 10, it clearly appears that the underestimation is mainly due to the error on the estimation of the critical Shields parameters. Indeed, the formulas induce an 
underestimation of the results when $\theta_{w g}<\theta_{c r}$ or $\theta_{w g}>\theta_{c r, s f}$, where $\theta_{c r}$ and $\theta_{c r, s f}$ are estimated values.

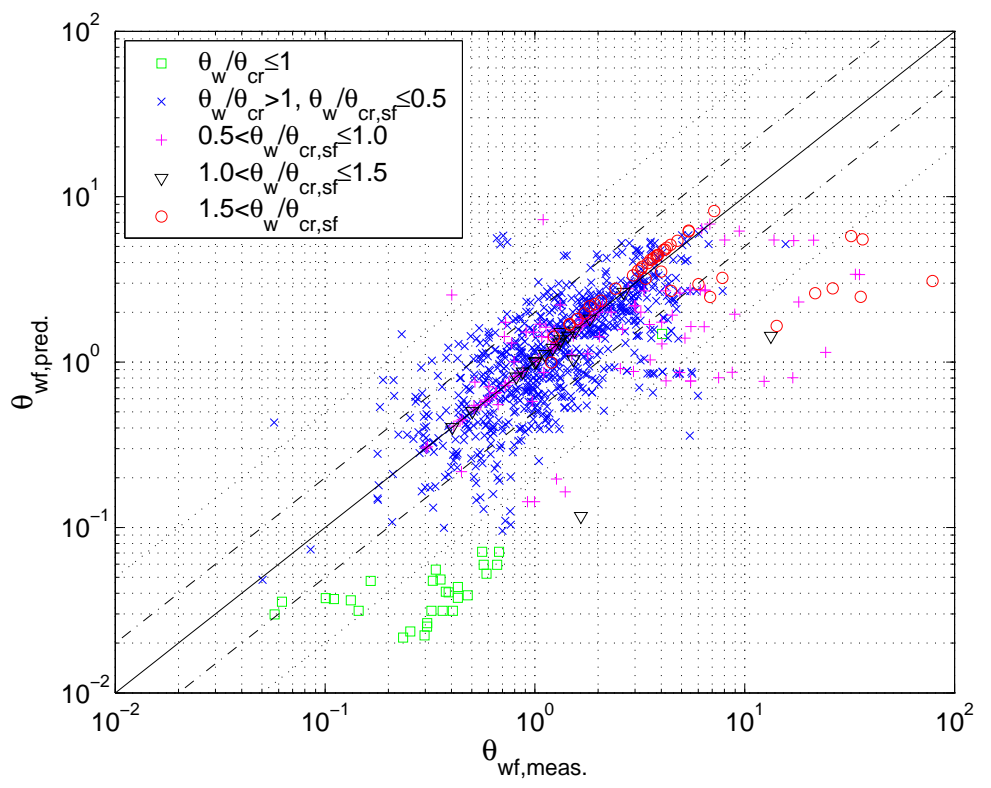

Figure 10: Prediction of the Shields parameter using Eq. 17 with $a_{r}=20$ for the estimation of the roughness and the observed and estimated (Eqs. 9 and 10 with $\left.\alpha_{c r}=1\right)$ characteristics of the ripples.

\section{Conclusion}

From this sensitivity analysis, it appears that large scatters exist in the prediction of ripple characteristics and so on the ripple-related roughness depending on the formulas applied. One important point to be considered is the critical Shields parameters for the inception of sediment movement $\theta_{c r}$ and for the inception of sheet flow $\theta_{c r, s f}$, which should border the limits for the existence of ripples. Only the Grant \& Madsen and Soulsby \& Whitehouse formulas take into account $\theta_{c r}$ and only the Nielsen and Van Rijn formulas include the sheet flow limit, even if both formulas seem to underestimate the wash-out of the ripples. Thus, even if the Soulsby \& Whitehouse formula yields the best prediction for the ripple characteristics and induced Shields parameter, it does not take into account properly the inception of sheet flow and largely overestimates the results when ripples are washed out.

A new set of formulas (Eqs. 9 to 13) for ripple characteristics was proposed based on these two critical Shields parameters. The ripple steepness $H_{r} / L_{r}$ and dimensionless ripple length $L_{r} / A_{w}$ were found to reach an equilibrium value as soon as $\theta_{c}<\theta_{w g}<\theta_{c r, s f}$ where $\theta_{c r, s f}$ is estimated based on Camenen \& Larson (2006) study. For the ripple steepness $H_{r} / L_{r}$, this equilibrium value appeared to be nearly constant and equal to 0.16 . For the dimensionless ripple length $L_{r} / A_{w}$, it has been found to be a decreasing function of $\chi$ and $\theta_{w g}$. 
It should be noted that this set of formulas does not take into account relict ripples. As suggested by Soulsby \& Whitehouse (2007?), it could be partly taken into account assuming ripples take pre-existing values of $H_{r}$ and $L_{r}$ when $\theta_{w}<\theta_{c r}$. However, the hysteresis effect due to relict ripples as observed by Traykovski et al. (1999) appears to be much more difficult to model, especially when there is a reorganisation from $2 \mathrm{D}$ to $3 \mathrm{D}$ wave ripples.

A discussion is also proposed to explain the differences observed for the case of large wave ripples (LWR). The effect of an additional current appeared not to be so significant for the prediction of ripple characteristics. However, it should strongly affect the induced roughness by smoothing the ripples and making the ripple system $3 \mathrm{D}$.

The importance of the critical Shields parameter appeared to be even more significant when calculating the total Shields parameter. The roughness height for a rippled bed was also found to be as sensitive to the parameter $a_{r}$ (which varies from 8 to 37.5 depending on the authors) as to the ripple height and length. One future challenge would be to properly estimate the coefficient $a_{r}$ depending on the shape of the ripples, and so on the ratio $\left|U_{c}\right| / U_{w}$, using both experimental data and numerical tests.

All the uncertainties to estimate these parameters might explain the large scattering observed in many results for the estimation of the sediment transport. A simple empirical formula (Eq. 19) was then suggested to estimate the total Shields parameter in the ripple regime directly from the grain-related Shields parameter. The excess shear stress is proportional to $a_{r}$ and a decreasing function of the ratio

$\theta_{w g} / \chi$. The proposed formula yields satisfactory results compared to the classical methods based on ripple characteristics.

\section{Acknowledgment}

This work was conducted under the Inlet Modeling System Work Unit of the Coastal Inlets Research Program, U.S. Army Corps of Engineers, and partly sponsored by the Japanese Society for the Promotion of Science. I also would like to thanks R.L. Soulsby for for his very helpful comments, suggestions, and corrections.

\section{References}

Andersen, K. (2001), The dissipation of waves over a rippled bed, in L. Van Rijn, A. Davies, J. Van der Graff \& J. Ribberink, eds, 'SEDMOC: Sediment transport Modelling in Marine Coastal Environments', Aqua Publications, The Netherlands, chapter AL.

Bagnold, R. (1946), 'Motion of waves in shallow water: interaction between waves and sand bottom', Proc. Royal Society of London A(187), 1-15. 
Bosman, J. (1982), Concentration distribution under waves and current, Technical Report M 1875, Coastal Eng. Dpt., Delft Univ. of Technology, Delft, The Netherlands. (in Dutch).

Camenen, B. \& Larson, M. (2006), 'Phase-lag effects in sheet flow transport', Coastal Eng. 53, 531-542.

Camenen, B. \& Larson, M. (2008), 'A suspended load sediment transport formula for the nearshore', J. Coastal Res. 24(3), 615-627.

Catano-Lopera, Y. \& Garcia, M. (2006a), 'Geometry and migration characteristics of bedforms under waves and currents. part 1: Sandwave morphodynamics', Coastal Eng. 53, 767-780.

Catano-Lopera, Y. \& Garcia, M. (2006b), 'Geometry and migration characteristics of bedforms under waves and currents. part 2: Ripples superimposed on sandwaves', Coastal Eng. 53, 781-792.

Delgado Blanco, M., Bell, P. \& Montaliu, J. (2004), A new look to the applicability of classical models for ripple prediction, in 'Proc. 29th Int. Conf. Coastal Eng.', ASCE, Lisbon, Portugal, pp. 1909-1921.

Dingler, J. (1974), Wave formed ripples in nearshore sands, $\mathrm{PhD}$ thesis, University of California, San Diego, California, USA.

Doucette, J. (2000), 'The distribution of nearshore bedforms and effects on sand suspension on low-energy, micro-tidal beaches in southwestern australia', Marine Geology 165, 41-61.

Doucette, J. (2002), 'Geometry and grain-size sorting of ripples on low-energy sandy beaches: field observations and model predictions', Sedimentology 49, 483-503.

Faraci, C. \& Foti, E. (2002), 'Geometry, migration and evolution of small-scale bedforms generated by regular and irregular waves', Coastal Eng. 47, 35-52.

Grant, W. \& Madsen, O. (1982), 'Movable bed roughness in unsteady oscillatory flow', J. Geophysical Res. 87(C1), 469-481.

Grasmeijer, B. \& Kleinhans, M. (2004), 'Observed and predicted bed forms and their effect on suspended sand concentrations', Coastal Eng. 51, 351-371.

Grasmeijer, B. \& Van Rijn, L. (1999), 'Transport of fine sands by currents and waves (iii)', J. Waterways, Port, Coastal \& Ocean Eng. 125(2), 71-79.

Hanes, D., Alymov, V. \& Chang, Y. (2001), 'Wave-formed ripples at Duck, North Carolina', J. Geophysical Res. 106(C10), 22575-22592.

Hanson, H. \& Camenen, B. (2007), Closed form solution for threshold velocity for initiation of sediment motion under waves, in 'Proc. Coastal Sediments'07', ASCE, New Orleans, Louisiana, USA, pp. 15-28. 
Hume, T., Green, M. \& Oldman, J. (1999), What happens at the seabed of a headland during tropical cyclone?, in 'Proc. Coastal Sediments'99', ASCE, Long Island, New York, USA, pp. 1836-1851.

Inman, D. (1957), Wave generated ripples in nearshore sands, Technical Report Tech. Memo. 100, Beach Erosion Board, US Army Corps of Engineers.

Jonsson, I. \& Carlsen, N. (1976), 'Experimental and theoretical investigations in a oscillatory turbulent boundary layer', J. Hydraulic Res. 14, 45-60.

Khelifa, A. \& Ouellet, Y. (2000), 'Prediction of sand ripple geometry under waves and current', J. Waterways, Port, Coastal $\&$ Ocean Eng. 126(1), 14-22.

Kim, H. (2004), 'Effective form roughness of ripples for waves', J. Coastal Res. 20(3), 731-738.

Kleinhans, M. (2005), Phase diagram of bed states in steady, unsteady, oscillatory and mixed flows, in L. Van Rijn, R. Soulsby, P. Hoekstra \& A. Davies, eds, 'SANDPIT: Sand Transport and Morphology of Offshore Sand Mining Pits', Aqua Publications, The Netherlands, chapter Q.

Lofquist, K. (1978), Sand ripple growth in an oscillatory-flow water tunnel, Technical Report TP-78-5, Coastal Engineering Research Center, U.S. Army Corps of Engineers. 101 pp.

Malarkey, J. \& Davies, A. (2003), 'A non-iterative procedure for the wiberg and harris (1994) oscillatory sand ripple predictor', J. Coastal Res. 19(3), 738-739.

Mathisen, P. \& Madsen, O. (1996), 'Waves and currents over a fixed ripples bed. 1. bottom roughness experienced by waves in the presence and absence of currents', J. Geophysical Res. 101(C7), 16533-16542.

Miller, R. \& Komar, P. (1980), 'A field investigation of the relationship between ripple spacing and near bottom water motions', J. Sedimentary Petrology 50(1), 183-191.

Mogridge, G., Davies, M. \& Willis, D. (1994), 'Geometry prediction for wavegenerated bedforms', Coastal Eng. 22, 255-286.

Nielsen, P. (1981), 'Dynamics and geometry of wave generated ripples', J. Geophysical Res. 86(C7), 6467-6472.

Nielsen, P. (1984), 'Field measurements of time-averaged suspended sediment concentrations under waves', Coastal Eng. 8, 51-72.

Nielsen, P. (1992), Coastal bottom boundary layers and sediment transport, Vol. 4 of Advanced Series on Ocean Engineering, World Scientific Publication, Singapore. 
O'Donoghue, T. \& Clubb, G. (2001), 'Sand ripples generated by regular oscillatory flow', Coastal Eng. 44, 101-115.

O’Donoghue, T., Doucette, J., Van der Werf, J. \& Ribberink, J. (2005), Flow tunnel measurements of full-scale ripples in oscillatory flow, in L. Van Rijn, R. Soulsby, P. Hoekstra \& A. Davies, eds, 'SANDPIT: Sans Transport and Morphology of Offshore Mining Pits', Aqua Publications, chapter V.

Ribberink, J. \& Al Salem, A. (1994), 'Sediment transport in oscillatory boundary layers in cases of rippled beds and sheet flow', J. Geophysical Res. 99(C6), 707727.

Sato, S. (1987), Oscillatory boundary layer flow and sand movement over ripples, PhD thesis, University of Tokyo, Tokyo, Japan.

Sleath, J. (1982), 'The suspension of sand by waves', J. Hydraulic Res. 20(5), 439451.

Sleath, J. \& Wallbridge, S. (2002), 'Pickup from ripples beds in oscillatory flow', J. Waterways, Port, Coastal $\&$ Ocean Eng. 128(6), 228-237.

Soulsby, R. (1997), Dynamics of marine sands, a manual for practical applications, Thomas Telford, London, UK. ISBN 0-7277-2584.

Soulsby, R. \& Whitehouse, R. (1997), Threshold of sediment motion in coastal environment, in 'Proc. Pacific Coasts and Ports'97 Conf.', University of Canterbury, Christchurch, New Zealand, pp. 149-154.

Soulsby, R. \& Whitehouse, R. (2005a), Prediction of ripple properties in shelf seas: Mark 1, predictor, Technical Report TR 150, HR Wallingford, Wallingford, UK.

Soulsby, R. \& Whitehouse, R. (2005b), Prediction of ripple properties in shelf seas: Mark 2, predictor for time evolution, Technical Report TR 154, HR Wallingford, Wallingford, UK.

Steetzel, H. (1985), Model tests of scour near the toe of dune revetments, Technical Report M 2051-Il, Coastal Eng. Dpt., Delft Univ. of Technology, Delft, The Netherlands. (in Dutch).

Swart, D. (1974), Offshore sediment transport and equilibrium beach profiles, Technical report, Delft Hydraulics Lab. Publ., Delft, The Netherlands.

Traykovski, P., Hay, A., Irish, J. \& Lynch, J. (1999), 'Geometry, migration, and evolution of wave orbital ripples at leo-15', J. Geophysical Res. 104(C1), 15051524 .

Van der Werf, J. \& Ribberink, J. (2004), Wave induced sediment transport processes in the ripple regime, in 'Proc. 29th Int. Conf. Coastal Eng.', ASCE, Lisbon, Portugal, pp. 1741-1753. 
Van der Werf, J., Ribberink, J., O’Donoghue, T. \& Doucette, J. (2006), 'Modelling and measurement of sand transport processes over full-scale ripples in oscillatory flow', Coastal Eng. 53, 657-673.

Van Rijn, L. (1993), Principles of sediment transport in rivers, estuaries and coastal seas, Aqua Publications, The Netherlands.

Van Rijn, L. (2007), 'Unified view of sediment transport by currents and waves. I: Initiation of motion, bed roughness, and bed-load transport', J. Hydraulic Eng. 133(6), 649-667.

Van Rijn, L., Davies, A., Van der Graff, J. \& Ribberink, J., eds (2001), Sedmoc: Sediment Transport Modelling in Marine Coastal Environments, Aqua Publications, Amsterdam, The Netherlands. ISBN 90-800346-4-5.

Van Rijn, L. \& Havinga, F. (1995), 'Transport of fine sands by currents and waves (II)', J. Waterways, Port, Coastal \& Ocean Eng. 121(2), 123-143.

Van Rijn, L., Nieuwjaar, M., Van der Kaay, T., Nap, E. \& Van Kampen, A. (1993), 'Transport of fine sands by currents and waves (I)', J. Waterways, Port, Coastal \& Ocean Eng. 119(2), 123-143.

Wiberg, P. \& Harris, C. (1994), 'Ripple geometry in wave-dominated environments', J. Geophysical Res. 99(C1), 775-789.

Wikramanayake, P. \& Madsen, O. (1991), Calculation of movable bed friction factors, Technical report, Massachusetts Inst. of Tech., Cambridge, Massachusetts. $105 \mathrm{pp}$.

Williams, J., Bell, P. \& Thorne, P. (2005), 'Unifying large and small wave-generated ripples', J. Geophysical Res. 110(C2008), 1-18.

Williams, J., Bell, P., Thorne, P., Metje, N. \& Coates, L. (2004), 'Measurement and prediction of wave-generated suborbital ripples', J. Geophysical Res. 109(C2004), 1-18.

Wilson, K. (1989), 'Friction of wave induced sheet flow', Coastal Eng. 12, 371-379.

\section{Appendix: Predictive equations for ripples charac- teristics}

\section{Nielsen formula (1981)}

Nielsen (1981) proposed the following equation for the ripple wave length based on the field data of Inman (1957), Dingler (1974), and Miller \& Komar (1980):

$$
L_{r}=\exp \left(\frac{693-0.37 \ln ^{8} \Psi_{w}}{1000+0.75 \ln ^{7} \Psi_{w}}\right) A_{w}
$$


Using the data by Inman (1957) and Dingler (1974), Nielsen (1981) observed a washout of the ripples for a skin Shields number $\theta_{w s} \approx 0.8-1$. The maximum ripple steepness also appeared larger for irregular waves $\left(\max \left(H_{r} / L_{r}\right) \approx 0.35\right)$ than regular waves $\left(\max \left(H_{r} / L_{r}\right) \approx 0.20\right)$. Nielsen proposed an equation for the ripple steepness in case of irregular waves:

$$
\frac{H_{r}}{L_{r}}=0.342-0.34 \theta_{w g}^{0.25}
$$

\section{Grant \& Madsen formula (1982)}

Grant \& Madsen (1982) presented a set of empirical equations for the prediction of ripple characteristics. They distinguished a break-off point (critical Shields parameter) below/above which the ripple steepness is increasing/decreasing:

$$
\theta_{B}=1.8 \theta_{c r}\left(\frac{d_{*}^{1.5}}{4}\right)^{0.6}
$$

They proposed the following relationships functions of the skin wave-related Shields parameter:

$$
\begin{aligned}
& \frac{H_{r}}{A_{w}}= \begin{cases}0.22\left(\frac{\theta_{w g}}{\theta_{c r}}\right)^{-0.16} & \text { for } \theta_{c r}<\theta_{w g} \leq \theta_{B} \\
0.48\left(\frac{d_{*}^{1.5}}{4}\right)^{0.8}\left(\frac{\theta_{w g}}{\theta_{c r}}\right)^{-1.5} & \text { for } \theta_{B}<\theta_{w g}\end{cases} \\
& \frac{H_{r}}{L_{r}}= \begin{cases}0.16\left(\frac{\theta_{w g}}{\theta_{c r}}\right)^{-0.04} & \text { for } \theta_{c r}<\theta_{w g} \leq \theta_{B} \\
0.28\left(\frac{d_{*}^{1.5}}{4}\right)^{0.6}\left(\frac{\theta_{w g}}{\theta_{c r}}\right)^{-1.0} & \text { for } \theta_{B}<\theta_{w g}\end{cases}
\end{aligned}
$$

\section{Van Rijn formula (1993)}

Van Rijn (1993) proposed the following relationships for the wave ripples in case of irregular waves and as functions of the mobility parameter $\Psi_{w}$ :

$$
\begin{aligned}
& \frac{H_{r}}{A_{w}}= \begin{cases}0.22 & \text { for } \Psi_{w} \leq 10 \\
2.8 \times 10^{-13}\left(250-\Psi_{w}\right)^{5} & \text { for } 10<\Psi_{w} \leq 250\end{cases} \\
& \frac{H_{r}}{L_{r}}= \begin{cases}0.18 & \text { for } \Psi_{w} \leq 10 \\
2.0 \times 10^{-7}\left(250-\Psi_{w}\right)^{2.5} & \text { for } 10<\Psi_{w} \leq 250\end{cases}
\end{aligned}
$$

\section{Wikramanayake \& Madsen formula (1991)}

Wikramanayake \& Madsen (1991) proposed a relationship using that the ratio of the mobility number $\Psi_{w}$ to the non dimensional sediment parameter $S_{*}$ :

$$
S_{*}=\frac{d_{50}}{4 \nu} \sqrt{(s-1) g d_{50}}
$$


For irregular waves, they chose $U_{r m s}$ to represent the wave orbital velocity $U_{w}$ (Assuming a Rayleigh distribution, $\left.U_{r m s}=1 / \sqrt{2} U_{1 / 3}\right)$. They found a good correlation with the field ripple data of Inman (1957), Dingler (1974) and Nielsen (1984) using the following equations:

$$
\begin{aligned}
& \frac{H_{r}}{A_{w}}= \begin{cases}0.27\left(\frac{\Psi_{w}}{S_{*}}\right)^{-0.5} & \text { for } \frac{\Psi_{w}}{S_{*}} \leq 3.0 \\
0.52\left(\frac{\Psi_{w}}{S_{*}}\right)^{-1.1} & \text { for } \frac{\Psi_{w}}{S_{*}}>3.0\end{cases} \\
& \frac{L_{r}}{A_{w}}= \begin{cases}1.70\left(\frac{\Psi_{w}}{S_{*}}\right)^{-0.5} & \text { for } \frac{\Psi_{w}}{S_{*}} \leq 3.0 \\
2.10\left(\frac{\Psi_{w}}{S_{*}}\right)^{-0.7} & \text { for } \frac{\Psi_{w}}{S_{*}}>3.0\end{cases}
\end{aligned}
$$

It should be noted that the coefficients have been modified as Wikramanayake \& Madsen (1991) based their results on the root-mean-square value of the wave excursion. Moreover, when $\Psi_{w} / S_{*} \leq 4.2$, the ripple height and wave length are only functions of the sediment characteristics.

\section{Mogridge et al. formula (1994)}

Mogridge et al. (1994) conducted an extensive study on previously published experimental data in both laboratory and field. They found some complex empirical relationships based on the wave period parameter $\chi$. The complexity of the formula makes it however difficult to use. A simplified version is as following:

$$
\begin{aligned}
& \frac{L_{r}}{d_{50}}=\min \left(10^{3.373-13.772 \chi^{0.02054}}, 1394\right) \\
& \frac{H_{r}}{d_{50}}=10^{8.542-10.822 \chi^{0.03967}}
\end{aligned}
$$

On a real case, the wave period and median grain size do not vary significantly along a cross-shore beach profile whereas ripple characteristics do vary a lot (from well formed ripples offshore to a flat bed in the surf zone as ripples are washed-out).

\section{Wiberg \& Harris formula (1994)}

Wiberg \& Harris (1994) proposed iterative formulas for the prediction of the ripple characteristics which is also difficult to use. However, Malarkey \& Davies (2003) proposed a simplified procedure to avoid the iterative calculation. The ripple characteristics read:

$$
L_{r}= \begin{cases}0.62 A_{w} & \text { for } A H<20 \\ 535 d_{50} \exp \left[-\ln \left(\frac{0.62 A_{w}}{535 d_{50}}\right) \frac{\ln (0.01 A H)}{\ln 5}\right] & \text { for } 20 \leq A H \leq 100 \\ 535 d_{50} & \text { for } A H>100\end{cases}
$$


with $A H=A_{w} / H_{r, \text { ano }}$ where $H_{r, a n o}$ is the anorbital ripple height calculated using the following equation for the ripple height with $L_{r}=535 d_{50}$ :

$$
\frac{A_{w}}{H_{r}}=\exp \left[C_{1}-\sqrt{C_{2}-C_{3} \ln \left(\frac{A_{w}}{L_{r}}\right)}\right]
$$

with $C_{1}=7.59, C_{2}=33.60$ and $C_{3}=10.53$.

\section{Grasmeijer \& Kleinhans formula (2004)}

More recently, Grasmeijer \& Kleinhans (2004) proposed some equations based on the Nielsen (1981) study. They fitted an empirical relationship using laboratory data by Van Rijn et al. (1993), Van Rijn \& Havinga (1995) and Grasmeijer \& Van Rijn (1999) and field data by Inman (1957), Hanes et al. (2001) as well as their own data set measured near the coast of Egmond aan Zee, The Netherlands:

$$
\begin{aligned}
& \frac{H_{r}}{A_{w}}= \begin{cases}0.275-0.022 \Psi_{w}{ }^{0.5} & \text { for } \Psi_{w} \leq 10 \\
2 \Psi_{w}{ }^{-1} & \text { for } 10<\Psi_{w}\end{cases} \\
& \frac{H_{r}}{L_{r}}= \begin{cases}-0.078+0.355 \Psi_{w}{ }^{-0.221} & \text { for } \Psi_{w} \leq 10 \\
0.14 & \text { for } 10<\Psi_{w}\end{cases}
\end{aligned}
$$

\section{Soulsby \& Whitehouse formula (2005)}

Soulsby \& Whitehouse (2005) proposed a new equation based on an extensive study and Wiberg \& Hanes (1994) observations. Following findings of O'Donoghue et al. (2005), for irregular waves, they chose $U_{1 / 10}$, the mean of the highest onetenth velocities to represent the wave orbital velocity $U_{w}$ (Assuming a Rayleigh distribution, $\left.U_{1 / 10}=1.80 U_{r m s}=1.27 U_{1 / 3}\right)$. In irregular waves, they assumed the peak-period $T_{p}$ gives the best representation of $T_{w}$.

$$
\begin{aligned}
& \frac{L_{r}}{A_{w}}=\left[1+1.87 \times 10^{-3} \frac{A_{w}}{d_{50}}\left(1-\exp \left\{-\left(2.0 \times 10^{-4} \frac{A_{w}}{d_{50}}\right)^{1.5}\right\}\right)\right]^{-1} \\
& \frac{H_{r}}{L_{r}}=0.15\left[1-\exp \left\{-\left(5000 \frac{d_{50}}{A_{w}}\right)^{3.5}\right\}\right]
\end{aligned}
$$

An additional constraint is that ripples can only form and evolve if the wave-induced stress exceeds the threshold of motion of the sediment, i.e. $\theta_{w g}>\theta_{c r}$. 